Cite this: Phys. Chem. Chem. Phys., 2014, 16, 10095

Received 29th October 2013, Accepted 31st January 2014

DOI: $10.1039 / c 3 c p 54569 e$

www.rsc.org/pccp

\title{
The 6Hankel asymptotic approximation for the uniform description of rainbows and glories in the angular scattering of state-to-state chemical reactions: derivation, properties and applications
}

\author{
Chengkui Xiahou and J. N. L. Connor*
}

\begin{abstract}
This paper considers the asymptotic (semiclassical) analysis of a forward glory and a rainbow in the differential cross section (DCS) of a state-to-state chemical reaction, whose scattering amplitude is given by a Legendre partial wave series (PWS). A recent paper by C. Xiahou, J. N. L. Connor and D. H. Zhang [Phys. Chem. Chem. Phys., 2011, 13, 12981] stated without proof a new asymptotic formula for the scattering amplitude, which is uniform for a glory and a rainbow in the DCS. The new formula was designated "6Hankel" because it involves six Hankel functions. This paper makes three contributions: (1) we provide a detailed derivation of the 6 Hankel approximation. This is done by first generalizing a method described by G. F. Carrier [J. Fluid Mech., 1966, 24, 641] for the uniform asymptotic evaluation of an oscillating integral with two real coalescing stationary phase points, which results in the "2Hankel" approximation (it contains two Hankel functions). Application of the 2 Hankel approximation to the PWS results in the 6 Hankel approximation for the scattering amplitude. We also test the accuracy of the 2 Hankel approximation when it is used to evaluate three oscillating integrals of the cuspoid type. (2) We investigate the properties of the 6 Hankel approximation. In particular, it is shown that for angles close to the forward direction, the 6Hankel approximation reduces to the "semiclassical transitional approximation" for glory scattering derived earlier. For scattering close to the rainbow angle, the 6 Hankel approximation reduces to the "transitional Airy approximation", also derived earlier. (3) Using a J-shifted Eckart parameterization for the scattering matrix, we investigate the accuracy of the 6Hankel approximation for a DCS. We also compare with angular scattering results from the "uniform Bessel", "uniform Airy" and other semiclassical approximations.
\end{abstract}

\section{Introduction}

The angular scattering of a state-to-state chemical reaction contains fundamental information on the dynamics and mechanism of the reaction. ${ }^{1}$ However, it has often proven difficult to quantitatively understand the physical content contained within a plot of the differential cross section (DCS) versus reactive scattering angle. Consider, for example, the $\mathrm{F}+\mathrm{H}_{2} \rightarrow \mathrm{FH}+\mathrm{H}$ reaction, whose angular scattering was measured in the famous crossed molecular-beam experiments of Neumark et al. ${ }^{2}$ in 1985. It took 19 years before the enhanced forward peak in the small-angle scattering of the product $\mathrm{FH}\left(v_{\mathrm{f}}=3\right)$ vibrational state was identified $^{3}$ as a glory. And it took 24 years before the scattering at larger angles in the DCS was recognized ${ }^{4}$ as a rainbow.

More recently (in 2008), state-of-the-art DCS measurements for the $\mathrm{F}+\mathrm{H}_{2}$ reaction were reported by Wang et al. ${ }^{5}$ using

School of Chemistry, The University of Manchester, Manchester M13 9PL, England, UK.E-mail:j.n.l.connor@manchester.ac.uk quantum-state-selected crossed molecular beams. An analysis of the angular scattering by ourselves and $\mathrm{Zhang}^{6}$ again revealed the presence of glories and rainbows in the $\mathrm{FH}\left(v_{\mathrm{f}}=3\right)$ DCSs; they are also accompanied by diffraction oscillations arising from nearside-farside interference.

The analyses in ref. 3, 4 and 6 used powerful asymptotic (semiclassical) techniques to extract physical information from the large number of interfering partial waves which contribute to the scattering amplitude. Two different asymptotic theories were employed: One theory ${ }^{3,7}$ led to the uniform glory approximation (and subsidiary approximations), whilst the second theory $^{8}$ resulted in the uniform (and transitional) rainbow approximations. A disadvantage of these theoretical treatments is that the uniform glory approximation becomes non-uniform for rainbow scattering, and vice versa. By a uniform approximation, we mean one in which the error remains approximately constant as a parameter, such as the reactive scattering angle, passes through certain critical values, such as zero (for a forward glory) or the rainbow angle. ${ }^{3,4,6-8}$ A transitional approximation is 
one in which the error remains small in the neighbourhood of these critical values, but the error usually increases as the parameter moves away from the critical values. ${ }^{3,4,6-8}$

It is desirable to develop new asymptotic scattering theories that are uniform for both forward glory and rainbow scattering. This was partially done in our paper with Zhang, ${ }^{6}$ where we stated without proof a new asymptotic approximation for the scattering amplitude that is valid for both a glory and a rainbow. We called our new result, the 6Hankel asymptotic approximation, since it contains six Hankel functions. ${ }^{6}$ For the DCS, our new result is a generalization to reactive scattering of a formula given by Miller $^{9}$ for elastic scattering.

The purpose of this paper is: (1) to present a derivation of the 6Hankel approximation, (2) to discuss its properties, and (3) to assess its accuracy for the DCS of a chemical reaction. In our DCS computations, we use a J-shifted Eckart parameterization of the scattering (S) matrix, ${ }^{10,11}$ as it allows flexibility in the location of the rainbow angle, thereby allowing us to test the 6 Hankel approximation over a wide angular range.

For information on the mathematical description of glories and rainbows, we refer to the extensive review by Adam. ${ }^{12}$ Earlier work on the role of forward glories in the DCSs of chemical reactions can be found in ref. 3, 6, 7, 10, 11 and 13-17. Likewise the role of rainbows is discussed in ref. 4 and 6 .

In order to describe a rainbow, we first must consider the uniform asymptotic evaluation of a one-dimensional oscillating integral with two coalescing stationary phase points. This is done in Section II. In particular, we generalize a method described by Carrier. ${ }^{18}$ He assumes that the phase of the integrand is an odd function of $x$ and that the pre-exponential factor is an even function of $x$, where $x$ is the integration variable. We extend his result to the case when neither of these symmetry properties holds. We call our generalization, the 2 Hankel asymptotic approximation, since it involves two Hankel functions. We also investigate the limit when the stationary phase points coalesce, which leads to the transitional Airy approximation ${ }^{4,6,8}$ for rainbow scattering.

Section III applies the 2 Hankel approximation to the scattering amplitude for a chemical reaction, when it is given by a Legendre partial wave series, thereby providing a derivation of the 6 Hankel approximation. The limit when the scattering angle tends to zero is investigated, resulting in the semiclassical transitional approximation ${ }^{3}$ previously derived for glory scattering.

The $J$-shifted Eckart parameterization for the $S$ matrix ${ }^{10,11}$ is defined in Section IV. The values of the parameters are chosen so that the rainbow angle occurs at a large value, namely $109.2^{\circ}$ in the centre-of-mass reference frame. This allows us to conduct a better test of the accuracy of the 6Hankel approximation than previously, which used numerical $\mathrm{S}$ matrix data. ${ }^{6}$ An important point is that the 6 Hankel formula is generic, i.e., it also applies to numerous chemical reactions at numerous different energies which have S matrix properties analogous to the $J$-shifted Eckart parameterization.

Section V describes our results for the DCS using the 6 Hankel and other semiclassical approximations. In order to provide additional physical insight into interference structure in the DCS, we have also applied nearside-farside (NF) theory ${ }^{19,20}$ and local angular momentum (LAM) theory, ${ }^{21-24}$ in both cases including up to three resummations of the partial wave series. ${ }^{21-24}$ We also make contact with complex angular momentum (or Regge pole) theory, which has been used to calculate DCSs. ${ }^{25-27}$

Our Conclusions are in Section VI. The Appendix describes a test of the 2 Hankel approximation when it is applied to three oscillating integrals of the cuspoid type. ${ }^{28,29}$

Finally, we emphasize that there is a long tradition in the chemical physics literature of papers concerned with the asymptotic evaluation of oscillating integrals, see for example ref. 3, 4, 6-17, 20, 23, 25-28 and 30-36.

\section{Uniform asymptotic integration: the 2Hankel approximation}

\section{IIA. Introduction}

This section is concerned with the uniform asymptotic evaluation of the oscillating integral:

$$
I(\alpha)=\int_{-\infty}^{\infty} g(x) \exp [\mathrm{i} f(\alpha ; x)] \mathrm{d} x
$$

where $\alpha$ is a real parameter and $f(\alpha ; x)$ is a real-valued function. In addition, $g(x)$ is slowly varying; it can be a real- or complexvalued function and may also depend on $\alpha$, although this is not indicated. We assume there exist two real points of stationary phase, denoted $x_{1}(\alpha)$ and $x_{2}(\alpha)$, with $x_{1}(\alpha) \leq x_{2}(\alpha)$, which are the roots of $f^{\prime}(\alpha ; x)=0$, where the prime indicates differentiation with respect to $x$. As $\alpha$ varies, it is assumed that the two roots coalesce on the caustic at $x=x\left(\alpha_{0}\right)$ for $\alpha=\alpha_{0}$.

The convenient notations $x_{i}=x_{i}(\alpha), g_{i}=g\left(x_{i}\right), f_{i}=f\left(\alpha ; x_{i}\right)$, $f_{i}^{\prime}=\left.f^{\prime}(\alpha ; x)\right|_{x=x_{i}}, f_{i}^{\prime \prime}=\left.f^{\prime \prime}(\alpha ; x)\right|_{x=x_{i}}$ and $f_{i}^{\prime \prime \prime}=\left.f^{\prime \prime \prime}(\alpha ; x)\right|_{x=x_{i}}$ for $i=0,1,2$ will often be employed in the following. In our application in Section III, $f(\alpha ; x)$ has a linear dependence on $\alpha$ of the type, $\pm \alpha x$, which means that the second and third derivatives of $f(\alpha ; x)$ are independent of $\alpha$. But note that $f_{i}^{\prime \prime}$ and $f_{i}^{\prime \prime \prime}$ do depend on $\alpha$ via the $x_{i}=x_{i}(\alpha)$.

Given the above assumptions, two cases arise, depending on whether $f(\alpha ; x) \rightarrow+\infty$ or $\rightarrow-\infty$ as $x \rightarrow+\infty$ [or equivalently, $f(\alpha ; x) \rightarrow-\infty$ or $\rightarrow+\infty$ as $x \rightarrow-\infty]$. These two cases are illustrated in Fig. 1, where $f(\alpha ; x)$ is given by simple polynomial functions. The black solid curves for $f(\alpha ; x)$ in Fig. 1 possess a local maximum and a local minimum. Also illustrated are the curves (blue solid) when the two stationary points have coalesced for $\alpha=\alpha_{0}$. The two cases have the following properties, which we use later:

Case A - see Fig. 1(a)

For $x_{1}<x_{2}$ so that $\alpha \neq \alpha_{0}$

$$
\begin{gathered}
f_{1}>f_{2}, f_{1}{ }^{\prime}=f_{2}{ }^{\prime}=0, \\
f_{1}{ }^{\prime \prime}<0, f_{2}{ }^{\prime \prime}>0, f_{1}{ }^{\prime \prime \prime}>0, f_{2}{ }^{\prime \prime \prime}>0
\end{gathered}
$$

For $x_{1}=x_{2}=x_{0}$ so that $\alpha=\alpha_{0}$

$$
f_{0}^{\prime}=0, f_{0}^{\prime \prime}=0, f_{0}^{\prime \prime \prime}>0
$$



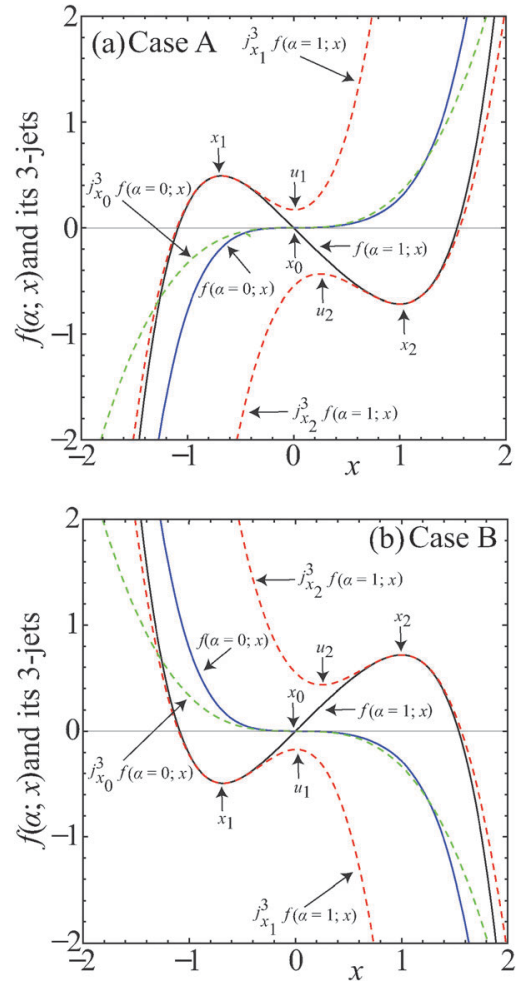

Fig. 1 Properties of the phase $f(\alpha ; x)$ and three of its 3 -jets. (a) Case A. Black solid line: $f(\alpha ; x)=-\alpha x+x^{3} / 3-x^{4} / 4+x^{5} / 5$ for $\alpha=1$. Its stationary phase points are given by, $x_{1} \approx-0.682328$ (local maximum) and $x_{2}=1$ (local minimum). Lower red dashed curve: The 3 -jet at $x_{2}, j_{x_{2}}{ }^{3} f(\alpha=1 ; x)$. Its stationary phase points are denoted, $u_{2}=1 / 4$ (local maximum) and $x_{2}=1$ (local minimum). Upper red dashed curve: The 3-jet at $x_{1}, j_{x_{1}}{ }^{3} f(\alpha=1 ; x)$. Its stationary phase points are denoted, $x_{1} \approx-0.682328$ (local maximum) and $u_{1} \approx 0.00804454$ (local minimum). Blue solid curve: $f(\alpha=0 ; x)=$ $x^{3} / 3-x^{4} / 4+x^{5} / 5$. Its two stationary phase points have coalesced at $x_{0}=0$. Green dashed curve: The 3 -jet at $x_{0}, j_{x_{0}}{ }^{3} f(\alpha=0 ; x)=x^{3} / 3$. Its two stationary phase points have coalesced at $x_{0}=0$. (b) Case B. Black solid line: $f(\alpha ; x)=$ $-\left(-\alpha x+x^{3} / 3-x^{4} / 4+x^{5} / 5\right)$ for $\alpha=1$. Its stationary phase points are given by, $x_{1} \approx-0.682328$ (local minimum) and $x_{2}=1$ (local maximum). Upper red dashed curve: The 3 -jet at $x_{2}, j_{x_{2}}{ }^{3} f(\alpha=1 ; x)$. Its stationary phase points are denoted, $u_{2}=1 / 4$ (local minimum) and $x_{2}=1$ (local maximum). Lower red dashed curve: The 3 -jet at $x_{1}, j_{x_{1}}{ }^{3} f(\alpha=1 ; x)$. Its stationary phase points are denoted, $x_{1} \approx-0.682328$ (local minimum) and $u_{1} \approx 0.00804454$ (local maximum). Blue solid curve: $f(\alpha=0 ; x)=-\left(x^{3} / 3-x^{4} / 4+x^{5} / 5\right)$. Its two stationary phase points have coalesced at $x_{0}=0$. Green dashed curve: The 3-jet at $x_{0}, j_{x_{0}}{ }^{3} f(\alpha=0 ; x)=$ $-x^{3} / 3$. Its two stationary phase points have coalesced at $x_{0}=0$.

Case B - see Fig. 1(b)

For $x_{1}<x_{2}$ so that $\alpha \neq \alpha_{0}$

$$
\begin{gathered}
f_{1}<f_{2}, f_{1}{ }^{\prime}={f_{2}}^{\prime}=0 \\
f_{1}^{\prime \prime}>0, f_{2}^{\prime \prime}<0, f_{1}^{\prime \prime \prime}<0, f_{2}{ }^{\prime \prime \prime}<0
\end{gathered}
$$

For $x_{1}=x_{2}=x_{0}$ so that $\alpha=\alpha_{0}$

$$
f_{0}{ }^{\prime}=0, f_{0}^{\prime \prime}=0, f_{0}{ }^{\prime \prime \prime}<0
$$

Remarks:

- It is also assumed that $g(x)$ does not possess any singularities or zeros near to the stationary phase points. A modified treatment can be given if this is not the case (e.g., see ref. 37 and 38, for example).

- If $f(\alpha ; x)=-f(\alpha ;-x)$, i.e., $f$ is an odd function of $x$, then $x_{1}=-x_{2}$.

- If $f(\alpha ; x)$ possesses more than two real stationary points, then the following derivation is valid locally in the region of the two coalescing stationary points, ${ }^{8,28}$ provided that the additional stationary points are well separated from the coalescing pair.

- The mathematical level of our derivations is similar to that of ref. 8 by one of us (JNLC) and Marcus (hereafter referred to as $\mathrm{CM}$ ). The $\mathrm{CM}$ paper applied a different technique, that of Chester, Friedman and Ursell, $^{39}$ for the uniform asymptotic evaluation of an oscillating integral with two coalescing saddle points. In particular, CM presented the Chester et al. technique in an accessible, yet general, way, making it straightforward to apply to problems in molecular scattering theory.

A comparison of Fig. 1(a) and (b), shows that Cases A and B are related by a minus sign. In the following, we focus only on Case A, since the uniform asymptotic theory for Case B is similar. We have chosen Case A because it is used in our analysis of a reactive DCS in Section III.

\section{IIB. Two limiting cases}

Before proceeding, it is helpful to write down results for $I(\alpha)$ in two limiting cases. The results we require can be found in CM. The first limiting case is when $x_{1}$ and $x_{2}$ are well separated. Then the simple stationary phase approximation (SPA) can be applied at both stationary points, which results in (from ref. 8)

$$
I_{\mathrm{SPA}}(\alpha)=g_{1} \sqrt{\frac{2 \pi}{\left|f_{1}{ }^{\prime \prime}\right|}} \exp \left[\mathrm{i}\left(f_{1}-\frac{\pi}{4}\right)\right]+g_{2} \sqrt{\frac{2 \pi}{f_{2}{ }^{\prime \prime}}} \exp \left[\mathrm{i}\left(f_{2}+\frac{\pi}{4}\right)\right]
$$

Notice that the simple stationary phase approximation (2) does not depend on $f_{i}^{\prime \prime \prime}$. Now in practice the sign of $f_{i}^{\prime \prime \prime}$ can change as $x_{1}$ and $x_{2}$ move far apart from $x_{0}$, i.e., the sign of $f_{i}^{\prime \prime \prime}$ is then no longer fixed by Cases A and B. We include this effect in our 2Hankel result in Section IIC.4. The simple stationary phase result (2) is equivalent to making a second-order Taylor series expansion of $f(\alpha ; x)$ at each stationary point, together with the approximations, $g(x) \approx g\left(x_{1}\right)$ and $g(x) \approx g\left(x_{2}\right)$.

The second limiting case is when $\alpha \approx \alpha_{0}$ so that $x_{1} \approx x_{0} \approx x_{2}$. We make a third-order Taylor series expansion of $f(\alpha ; x)$ at the point $x_{0}$

$$
f(\alpha ; x) \approx f\left(\alpha ; x_{0}\right)+f^{\prime}\left(\alpha ; x_{0}\right)\left(x-x_{0}\right)+\frac{1}{6} f^{\prime \prime \prime}\left(\alpha ; x_{0}\right)\left(x-x_{0}\right)^{3}
$$

where the result $f^{\prime \prime}\left(\alpha ; x_{0}\right)=0$ has been used. Then making the approximation, $g(x) \approx g\left(x_{0}\right)$, we obtain the transitional Airy approximation (tAiry), namely (see ref. 8)

$$
I_{\text {tAiry }}(\alpha)=2 \pi g_{0} \exp \left(\mathrm{i} f_{0}\right)\left(\frac{2}{f_{0}{ }^{\prime \prime \prime}}\right)^{1 / 3} \operatorname{Ai}\left(f_{0}{ }^{\prime}\left(\frac{2}{f_{0}{ }^{\prime \prime \prime}}\right)^{1 / 3}\right)
$$


where $\operatorname{Ai}(\bullet)$ is the regular Airy function. The result (4) is valid for $f_{0}{ }^{\prime}<0$ (two real roots) as well as for $f_{0}{ }^{\prime}>0$ (two complex roots). When $\alpha=\alpha_{0}$, i.e., on the caustic, we have $f_{0}{ }^{\prime}=f^{\prime}\left(\alpha_{0} ; x_{0}\right)=0$, and eqn (4) simplifies to

$$
\begin{aligned}
I_{\text {tAiry }}\left(\alpha_{0}\right) & =2 \pi g_{0} \exp \left(\mathrm{i} f_{0}\right)\left(\frac{2}{f_{0}{ }^{\prime \prime \prime}}\right)^{1 / 3} \operatorname{Ai}(0) \\
& =\frac{2 \pi}{3^{2 / 3} \Gamma(2 / 3)} g_{0} \exp \left(\mathrm{i} f_{0}\right)\left(\frac{2}{f_{0}{ }^{\prime \prime \prime}}\right)^{1 / 3}
\end{aligned}
$$

where $\Gamma(\bullet)$ is the Gamma function.

In the following, to avoid repeating phrases like "the $k$ th-order Taylor series expansion of $f(\alpha ; x)$ at the point $x_{i}$ ", we will often use the concept of a $k$-jet, ${ }^{40,41}$ which is defined as the Taylor series expansion of order $k$ for $f(\alpha ; x)$ at the point $x_{i}$. For example, eqn (3) written as a 3 -jet is

$$
j_{x_{0}}{ }^{3} f(\alpha ; x)=f\left(\alpha ; x_{0}\right)+f^{\prime}\left(\alpha ; x_{0}\right)\left(x-x_{0}\right)+\frac{1}{6} f^{\prime \prime \prime}\left(\alpha ; x_{0}\right)\left(x-x_{0}\right)^{3}
$$

Note: all $k$-jets in this paper are functions and not members of a polynomial ring.

The problem now is to deduce a uniform approximation which reduces to eqn (2) and (4) in the appropriate limits.

\section{IIC. Extension of Carrier's method}

IIC.1 Introduction. Carrier presents his derivation for the integral $I(\alpha)$ in the Appendix of his paper, ${ }^{18}$ assuming that $f(\alpha ; x)$ is an odd function of $x$, and for $g(x)=1(n . b$. , there are many misprints). At the end of his derivation, he extends his result assuming $g(x)$ to be an even function of $x$. We need to generalize Carrier's derivation to the case when neither of these assumptions is valid.

The basic idea of Carrier is to use 3 -jets at the points $x_{1}$ and $x_{2}$, similar to eqn (3) or (6) for the point $x_{0}$. This seems straightforward and physically reasonable, but Fig. 1(a) shows there is a serious problem. We see that the 3 -jet at $x_{2}$ is accurate close to $x_{2}$, but it possesses another stationary point, say $u_{2}=u_{2}(\alpha)$, which is quite different from $x_{1}$. This implies that the approximation to $I(\alpha)$ using the 3 -jet at $x_{2}$, denoted $I_{x_{2}}(\alpha)$, will be quite different from $I(\alpha)$, unless $f(\alpha ; x)$ and $j_{x_{2}}{ }^{3} f(\alpha ; x)$ are very similar as functions of $x$. Evidently we must eliminate the contribution from the additional stationary point $u_{2}$.

Similar remarks apply if the 3 -jet at $x_{1}$ is used - see Fig. 1(a); we must eliminate the contribution from the additional stationary point $u_{1}$. And similarly for Case B in Fig. 1(b). Eliminating the unwanted contributions from $u_{1}$ and $u_{2}$ is the essence of our derivation.

IIC. 2 Contribution to $I(\alpha)$ from a third-order Taylor expansion at the stationary point $x_{2}$. The 3 -jet at $x_{2}$ is given by

$$
j_{x_{2}}{ }^{3} f(\alpha ; x)=f_{2}+\frac{1}{2} f_{2}{ }^{\prime \prime}\left(x-x_{2}\right)^{2}+\frac{1}{6}{f_{2}}^{\prime \prime \prime}\left(x-x_{2}\right)^{3}
$$

using $f_{2}{ }^{\prime}=0$. Then assuming $g(x) \approx g\left(x_{2}\right)$, we obtain an approximation denoted $I_{x_{2}}(\alpha)$ to $I(\alpha)$, namely

$I_{x_{2}}(\alpha)=g_{2} \exp \left(\mathrm{i} f_{2}\right) \int_{-\infty}^{\infty} \exp \left\{\mathrm{i}\left[\frac{1}{2} f_{2}^{\prime \prime}\left(x-x_{2}\right)^{2}+\frac{1}{6} f_{2}{ }^{\prime \prime \prime}\left(x-x_{2}\right)^{3}\right]\right\} \mathrm{d} x$

where the integration limits have been kept at $-\infty$ to $+\infty$. Denoting the stationary phase points of the integrand in eqn (8) by $x_{2}$ and $u_{2}$ as in Fig. 1 (a) with $u_{2} \leq x_{2}$, we find

$$
\begin{aligned}
& x_{2} \text { (by construction) } \\
& u_{2}=x_{2}-\left(2 f_{2}^{\prime \prime} / f_{2}^{\prime \prime \prime}\right)
\end{aligned}
$$

Next we use the identity

$\int_{-\infty}^{\infty} \mathrm{d} x \exp \left[\mathrm{i}\left( \pm \frac{a}{6} x^{3}+\frac{b}{2} x^{2}\right)\right]=2 \pi\left(\frac{2}{|a|}\right)^{1 / 3} \exp (\mathrm{i} \varsigma) \mathrm{Ai}\left(-\left(\frac{3}{2^{\varsigma}}\right)^{2 / 3}\right)$

where

$$
\varsigma=b^{3} /\left(3 a^{2}\right)
$$

which is valid for $a<0$ or $a>0$ but $a \neq 0$, and $b \leq 0$ or $b>0$. When $b<0$, the argument of the Airy function is to be interpreted as $-b^{2} /\left(2 a^{2}\right)^{2 / 3}$. Applying eqn (11) to eqn (8) gives

$$
I_{x_{2}}(\alpha)=2 \pi g_{2}\left(\frac{2}{\left|f_{2}{ }^{\prime \prime \prime}\right|}\right)^{1 / 3} \exp \left[\mathrm{i}\left(f_{2}+A_{2}\right)\right] \mathrm{Ai}\left(-\left(\frac{3}{2} A_{2}\right)^{2 / 3}\right)
$$

where

$$
A_{2}=\frac{\left(f_{2}^{\prime \prime}\right)^{3}}{3\left(f_{2}^{\prime \prime \prime}\right)^{2}}
$$

The result (12) evidently contains contributions from both the stationary points, $x_{2}$ and $u_{2}$.

Next we use the following identity connecting $\operatorname{Ai}(\bullet)$ and Hankel functions of the first and second kinds, $H_{1 / 3}^{(1)}(\bullet)$ and $H_{1 / 3}^{(2)}(\bullet)$ respectively, both of order one-third. ${ }^{42}$

$$
\operatorname{Ai}(-z)=\frac{1}{2} \sqrt{\frac{z}{3}}\left[\exp \left(\frac{\mathrm{i} \pi}{6}\right) H_{1 / 3}^{(1)}(\xi)+\exp \left(-\frac{\mathrm{i} \pi}{6}\right) H_{1 / 3}^{(2)}(\xi)\right]
$$

where

$$
\xi=\frac{2}{3} z^{3 / 2} \text { or equivalently, } z=\left(\frac{3}{2} \xi\right)^{2 / 3}
$$

We now use the identity (14) to write eqn (12) as the sum of two terms

$$
I_{x_{2}}(\alpha)=I_{x_{2}}^{(1)}(\alpha)+I_{x_{2}}^{(2)}(\alpha)
$$

where

$$
I_{x_{2}}^{(1)}(\alpha)=\frac{\pi}{3^{1 / 6}} g_{2}\left(\frac{A_{2}}{\left|f_{2}^{\prime \prime \prime}\right|}\right)^{1 / 3} \exp \left[\mathrm{i}\left(f_{2}+A_{2}+\frac{\pi}{6}\right)\right] H_{1 / 3}^{(1)}\left(A_{2}\right)
$$

and

$$
I_{x_{2}}^{(2)}(\alpha)=\frac{\pi}{3^{1 / 6}} g_{2}\left(\frac{A_{2}}{\left|f_{2}^{\prime \prime \prime}\right|}\right)^{1 / 3} \exp \left[\mathrm{i}\left(f_{2}+A_{2}-\frac{\pi}{6}\right)\right] H_{1 / 3}^{(2)}\left(A_{2}\right)
$$


Next we consider the limit where $x_{2}$ and $u_{2}$ are well separated, so we can use the following asymptotic approximations for the Hankel functions ${ }^{43}$ as $x \rightarrow \infty$

$$
\begin{aligned}
H_{\nu}^{(1)}(x) & \sim \sqrt{\frac{2}{\pi x}} \exp \left[\mathrm{i}\left(x-\frac{1}{2} \pi \nu-\frac{\pi}{4}\right)\right] \\
H_{\nu}^{(2)}(x) & \sim \sqrt{\frac{2}{\pi x}} \exp \left[-\mathrm{i}\left(x-\frac{1}{2} \pi \nu-\frac{\pi}{4}\right)\right]
\end{aligned}
$$

with $\nu=1 / 3$. We obtain for $I_{x_{2}}^{(1)}(\alpha)$ and $I_{x_{2}}^{(2)}(\alpha)$ the results

$$
\begin{gathered}
I_{x_{2}}^{(1)}(\alpha)=g_{2} \sqrt{\frac{2 \pi}{f_{2}^{\prime \prime}}} \exp \left[\mathrm{i}\left(f_{2}+2 A_{2}-\frac{\pi}{4}\right)\right] \\
I_{x_{2}}^{(2)}(\alpha)=g_{2} \sqrt{\frac{2 \pi}{f_{2}^{\prime \prime}}} \exp \left[\mathrm{i}\left(f_{2}+\frac{\pi}{4}\right)\right]
\end{gathered}
$$

Inspection of eqn (20) and (21) shows that $I_{x_{2}}^{(2)}(\alpha)$ is the simple stationary phase result for $x_{2}$, as given by the second term on the rhs of eqn (2). This suggests the other integral, $I_{x_{2}}^{(1)}(\alpha)$, is associated with the simple stationary phase result at $u_{2}$. We now confirm this suggestion by expressing $f_{2}, A_{2}$ and $f_{2}{ }^{\prime \prime}$ in eqn (20) in terms of $u_{2}$ rather than $x_{2}$.

From eqn (7), we have for $x=u_{2}$

$$
j_{x_{2}}{ }^{3} f\left(\alpha ; u_{2}\right)=f_{2}+\frac{1}{2} f_{2}^{\prime \prime}\left(u_{2}-x_{2}\right)^{2}+\frac{1}{6} f_{2}^{\prime \prime \prime}\left(u_{2}-x_{2}\right)^{3}
$$

which simplifies to

$$
j_{x_{2}}{ }^{3} f\left(\alpha ; u_{2}\right)=f_{2}+2 A_{2}
$$

when eqn (10) is used and $A_{2}$ is defined by eqn (13). Also from eqn (7) we have upon differentiation

$$
\frac{\mathrm{d}^{2} j_{x_{2}}{ }^{3} f(\alpha ; x)}{\mathrm{d} x^{2}}=f_{2}^{\prime \prime}+{f_{2}}^{\prime \prime \prime}\left(x-x_{2}\right)
$$

and so for $x=u_{2}$

$$
\left.\frac{\mathrm{d}^{2} j_{x_{2}}{ }^{3} f(\alpha ; x)}{\mathrm{d} x^{2}}\right|_{x=u_{2}}=f_{2}^{\prime \prime}+f_{2}^{\prime \prime \prime}\left(u_{2}-x_{2}\right)
$$

which simplifies to

$$
\left.\frac{\mathrm{d}^{2} j_{x_{2}}{ }^{3} f(\alpha ; x)}{\mathrm{d} x^{2}}\right|_{x=u_{2}}=-f_{2}^{\prime \prime}
$$

upon using eqn (10). With the help of eqn (22) and (23), we can now write eqn (20) in the form

$I_{x_{2}}^{(1)}(\alpha)=g_{2} \sqrt{2 \pi /\left(-\left.\frac{\mathrm{d}^{2} j_{x_{2}}{ }^{3} f(\alpha ; x)}{\mathrm{d} x^{2}}\right|_{x=u_{2}}\right)} \exp \left[\mathrm{i}\left(j_{x_{2}}{ }^{3} f\left(\alpha ; u_{2}\right)-\frac{\pi}{4}\right)\right]$

Eqn (24) is the simple stationary phase result at $u_{2}$ for $j_{x_{2}}{ }^{3} f(\alpha ; x)$, except for the presence of $g_{2} \equiv g\left(x_{2}\right)$ rather than $g\left(u_{2}\right)$. However this small difference is of no consequence because it is $I_{x_{2}}^{(1)}(\alpha)$ that we must eliminate from our theory, since eqn (24) may be quite different from the simple stationary phase result at $x_{1}$ for $I(\alpha)$, as given by the first term on the rhs of eqn (2), namely

$$
g_{1} \sqrt{\frac{2 \pi}{\left|f_{1}^{\prime \prime}\right|}} \exp \left[\mathrm{i}\left(f_{1}-\frac{\pi}{4}\right)\right]
$$

Note: The fact that $f(\alpha ; x)$ has a maximum at $x=x_{1}$ did not play an essential role in the argument that $I_{x_{2}}^{(2)}(\alpha)$ should be retained and $I_{x_{2}}^{(1)}(\alpha)$ discarded when $f(\alpha ; x)$ is approximated by $j_{x_{2}}{ }^{3} f(\alpha ; x)$. Thus if $f(\alpha ; x)$ increases monotonically for $x<x_{2}$, we would still retain $I_{x_{2}}^{(2)}(\alpha)$ and discard $I_{x_{2}}^{(1)}(\alpha)$ when using $j_{x_{2}}{ }^{3} f(\alpha ; x)$. Also, if in eqn (1), $g(x)=$ constant, then trivially $g\left(x_{2}\right)=g\left(u_{2}\right)$.

In the next section, we repeat the above analysis using the 3 -jet at $x_{1}$ rather than $x_{2}$.

IIC.3 Contribution to $I(\alpha)$ from a third-order Taylor expansion at the stationary point $\boldsymbol{x}_{1}$. The following derivation is analogous to that presented in Section IIC.2 and is shorter because only an outline is presented. Note that eqn (27)-(30) derived below are used in Sections IIC. 4 and IIIC.

The 3 -jet at $x_{1}$ is given by

$$
j_{x_{1}}^{3} f(\alpha ; x)=f_{1}-\frac{1}{2}\left|f_{1}^{\prime \prime}\right|\left(x-x_{1}\right)^{2}+\frac{1}{6} f_{1}^{\prime \prime \prime}\left(x-x_{1}\right)^{3}
$$

using $f_{1}{ }^{\prime}=0$. Then assuming $g(x) \approx g\left(x_{1}\right)$, we get for the approximation, $I_{x_{1}}(\alpha)$ to $I(\alpha)$, the integral

$I_{x_{1}}(\alpha)$

$$
=g_{1} \exp \left(\mathrm{i} f_{1}\right) \int_{-\infty}^{\infty} \exp \left\{\mathrm{i}\left[-\frac{1}{2}\left|f_{1}{ }^{\prime \prime}\right|\left(x-x_{1}\right)^{2}+\frac{1}{6} f_{1}{ }^{\prime \prime \prime}\left(x-x_{1}\right)^{3}\right]\right\} \mathrm{d} x
$$

with the integration limits kept at $-\infty$ to $+\infty$. Denoting the stationary phase points for the integrand of the integral (26) by $x_{1}$ and $u_{1}$ with $x_{1} \leq u_{1}$ [see Fig. $1(\mathrm{a})$ ], we have

$u_{1}=x_{1}+\left(2\left|f_{1}^{\prime \prime}\right| / f_{1}{ }^{\prime \prime \prime}\right)$ and $x_{1}$ (by construction). Next we apply the identity (11) to eqn (26) obtaining

$$
I_{x_{1}}(\alpha)=2 \pi g_{1}\left(\frac{2}{\left|f_{1}{ }^{\prime \prime \prime}\right|}\right)^{1 / 3} \exp \left[\mathrm{i}\left(f_{1}-A_{1}\right)\right] \mathrm{Ai}\left(-\left(\frac{3}{2} A_{1}\right)^{2 / 3}\right)
$$

where

$$
A_{1}=\frac{\left|f_{1}^{\prime \prime}\right|^{3}}{3\left(f_{1}{ }^{\prime \prime \prime}\right)^{2}}
$$

The result (27) evidently contains contributions from both $x_{1}$ and $u_{1}$.

We again use the relations (14) and (15) connecting $\operatorname{Ai}(\bullet)$ and the Hankel functions of the first and second kinds. This lets write eqn (27) as the sum of two terms

$$
I_{x_{1}}(\alpha)=I_{x_{1}}^{(1)}(\alpha)+I_{x_{1}}^{(2)}(\alpha)
$$

where

$$
I_{x_{1}}^{(1)}(\alpha)=\frac{\pi}{3^{1 / 6}} g_{1}\left(\frac{A_{1}}{\left|f_{1}^{\prime \prime \prime}\right|}\right)^{1 / 3} \exp \left[\mathrm{i}\left(f_{1}-A_{1}+\frac{\pi}{6}\right)\right] H_{1 / 3}^{(1)}\left(A_{1}\right)
$$


and

$$
I_{x_{1}}^{(2)}(\alpha)=\frac{\pi}{3^{1 / 6}} g_{1}\left(\frac{A_{1}}{\left|f_{1}^{\prime \prime \prime}\right|}\right)^{1 / 3} \exp \left[\mathrm{i}\left(f_{1}-A_{1}-\frac{\pi}{6}\right)\right] H_{1 / 3}^{(2)}\left(A_{1}\right)
$$

Next we consider the limit where $x_{1}$ and $u_{1}$ are sufficiently well separated that we can use the asymptotic approximations for the Hankel functions, as given by eqn (18) and (19) for $x \rightarrow \infty$ with $\nu=1 / 3$. We obtain

$$
\begin{gathered}
I_{x_{1}}^{(1)}(\alpha)=g_{1} \sqrt{\frac{2 \pi}{\left|f_{1}{ }^{\prime \prime}\right|}} \exp \left[\mathrm{i}\left(f_{1}-\frac{\pi}{4}\right)\right] \\
I_{x_{1}}^{(2)}(\alpha)=g_{1} \sqrt{\frac{2 \pi}{\left|f_{1}{ }^{\prime \prime}\right|}} \exp \left[\mathrm{i}\left(f_{1}-2 A_{1}+\frac{\pi}{4}\right)\right]
\end{gathered}
$$

Inspection of eqn (31) and (32) shows that $I_{x_{1}}^{(1)}(\alpha)$ is the simple stationary phase result at $x_{1}$, given by the first term on the rhs of eqn (2). Hence $I_{x_{1}}^{(2)}(\alpha)$ should be associated with the simple stationary phase result at $u_{1}$. We confirm this suggestion by manipulations in which $f_{1}, A_{1}$ and $f_{1}^{\prime \prime}$ in eqn (32) are expressed in terms of $u_{1}$ rather than $x_{1}$-these manipulations are analogous to those in Section IIC.2 for eqn (20). It is found that $I_{x_{1}}^{(2)}(\alpha)$ can be written in the alternative form

$$
I_{x_{1}}^{(2)}(\alpha)=g_{1} \sqrt{2 \pi /\left.\frac{\mathrm{d}^{2} j_{x_{1}}{ }^{3} f(\alpha ; x)}{\mathrm{d} x^{2}}\right|_{x=u_{1}}} \exp \left[\mathrm{i}\left(j_{x_{1}}{ }^{3} f\left(\alpha ; u_{1}\right)+\frac{\pi}{4}\right)\right]
$$

Now eqn (33) is the simple stationary phase result for $j_{x_{1}}{ }^{3} f(\alpha ; x)$ at $u_{1}$, except for the presence of $g_{1} \equiv g\left(x_{1}\right)$ rather than $g\left(u_{1}\right)$. Again this small difference is of no consequence since it is $I_{x_{1}}^{(2)}(\alpha)$ that we must eliminate from our theory. Note that eqn (33) may be quite different from the simple stationary phase result at $x_{2}$ for $I(\alpha)$, which is given by the second term on the rhs of eqn (2), namely

$$
g_{2} \sqrt{\frac{2 \pi}{f_{2}^{\prime \prime}}} \exp \left[\mathrm{i}\left(f_{2}+\frac{\pi}{4}\right)\right]
$$

Note: The fact that $f(\alpha ; x)$ has a minimum at $x=x_{2}$ did not play an essential role in the argument that $I_{x_{1}}^{(1)}(\alpha)$ should be retained and $I_{x_{1}}^{(2)}(\alpha)$ discarded when $f(\alpha ; x)$ is approximated by $j_{x_{1}}{ }^{3} f(\alpha ; x)$. Thus if $f(\alpha ; x)$ decreases monotonically for $x>x_{1}$, we would still retain $I_{x_{1}}^{(1)}(\alpha)$ and discard $I_{x_{1}}^{(2)}(\alpha)$ when using $j_{x_{1}}{ }^{3} f(\alpha ; x)$. This observation is used in Section IIIC for the nearside scattering of the $J$-shifted Eckart parametrization of the S matrix. Also, if in eqn (1), $g(x)=$ constant, then trivially $g\left(x_{1}\right)=g\left(u_{1}\right)$.

IIC.4 2Hankel approximation to $\boldsymbol{I}(\boldsymbol{\alpha})$. The 2Hankel approximation is obtained by retaining $I_{x_{1}}^{(1)}(\alpha)$ and $I_{x_{2}}^{(2)}(\alpha)$ [i.e., eqn (29) and (17) respectively]. But we drop $I_{x_{1}}^{(2)}(\alpha)$ and $I_{x_{2}}^{(1)}(\alpha)$ [i.e., eqn (30) and (16) respectively] because they correspond to unwanted contributions from the stationary phase points $u_{1}$ and $u_{2}$ respectively, which arise when $f(\alpha ; x)$ is approximated by its 3-jet at $x_{1}$ and $x_{2}$ respectively.

The 2 Hankel approximation, denoted $I_{2 \mathrm{H}}(\alpha)$, is thus given by

$$
I_{2 \mathrm{H}}(\alpha)=I_{x_{1}}^{(1)}(\alpha)+I_{x_{2}}^{(2)}(\alpha)
$$

where

$$
\begin{gathered}
I_{x_{1}}^{(1)}(\alpha)=\frac{\pi}{3^{1 / 6}} g_{1}\left(\frac{A_{1}}{\left|f_{1}{ }^{\prime \prime \prime}\right|}\right)^{1 / 3} \exp \left[\mathrm{i}\left(f_{1}-A_{1}+\frac{\pi}{6}\right)\right] H_{1 / 3}^{(1)}\left(A_{1}\right) \\
\sim g_{1} \sqrt{\frac{2 \pi}{\left|f_{1}{ }^{\prime \prime}\right|}} \exp \left[\mathrm{i}\left(f_{1}-\frac{\pi}{4}\right)\right]
\end{gathered}
$$

with

$$
A_{1}=\frac{\left|f_{1}^{\prime \prime}\right|^{3}}{3\left(f_{1}^{\prime \prime \prime}\right)^{2}}
$$

and

$$
\begin{gathered}
I_{x_{2}}^{(2)}(\alpha)=\frac{\pi}{3^{1 / 6}} g_{2}\left(\frac{A_{2}}{\left|f_{2}^{\prime \prime \prime}\right|}\right)^{1 / 3} \exp \left[\mathrm{i}\left(f_{2}+A_{2}-\frac{\pi}{6}\right)\right] H_{1 / 3}^{(2)}\left(A_{2}\right) \\
\sim g_{2} \sqrt{\frac{2 \pi}{f_{2}^{\prime \prime}}} \exp \left[\mathrm{i}\left(f_{2}+\frac{\pi}{4}\right)\right]
\end{gathered}
$$

with

$$
A_{2}=\frac{\left(f_{2}^{\prime \prime}\right)^{3}}{3\left(f_{2}^{\prime \prime \prime}\right)^{2}}
$$

Remarks:

- The 2Hankel approximation has the advantage that the two stationary phase points, $x_{1}$ and $x_{2}$, appear separately in eqn (34)-(40).

- The 2Hankel approximation has the disadvantage that it involves the third derivatives, $f_{1}^{\prime \prime \prime}$ and $f_{2}^{\prime \prime \prime}$. For numerical input data with associated errors, these third derivatives may be difficult to determine accurately.

- Note that the asymptotic limits (36) and (39), valid when $x_{1}$ and $x_{2}$ are well separated, do not involve third derivatives. Typically, $H_{1 / 3}^{(1)}(x)$ and $H_{1 / 3}^{(2)}(x)$ attain their asymptotic limits in eqn (18) and (19) respectively for $x \gtrsim 1$.

- As $x \rightarrow 0$, we have $H_{1 / 3}^{(1)}(x) \rightarrow-\mathrm{i} \infty$ and $H_{1 / 3}^{(2)}(x) \rightarrow \mathrm{i} \infty$, or in more detail (from ref. 44)

$$
H_{1 / 3}^{(1,2)}(x) \rightarrow \mp\left(\frac{\mathrm{i}}{\pi}\right) \Gamma\left(\frac{1}{3}\right)\left(\frac{2}{x}\right)^{1 / 3}
$$

where $\Gamma(\bullet)$ is a gamma function. These results imply that the 2 Hankel expression (34) becomes numerically unstable as $A_{1}$ and $A_{2}$ approach zero because of subtractive cancellation. This can be a problem for numerical input data. In Section IIC.5, we investigate analytically the 2 Hankel limit when $A_{1} \rightarrow 0$ and $A_{2} \rightarrow 0$.

- In practical applications of the 2 Hankel approximation, the third derivatives can change sign as $\alpha$ varies; in particular, as $x_{1}$ and $x_{2}$ separate and we approach the stationary phase 
results (36) and (39). Eqn (34)-(40) have been written so they are valid for either sign [If the third derivatives are identically zero, then eqn (34), (35), (37), (38) and (40) are ill-defined].

- If $g(x)=1$ and $f(\alpha ; x)$ is exactly a cubic polynomial in $x$, eqn (4), (12), (27) and the 2Hankel formula (34) are exact results for $I(\alpha)$ because the 3 -jets are then exact representations of $f(\alpha ; x)$. This is useful for the checking of computer programs.

Since our application of the 2Hankel approximation to reactive scattering in Section III is relatively complicated, in the Appendix we apply the 2 Hankel approximation to three oscillating integrals of the cuspoid type. ${ }^{28,29}$

IIC.5 Limiting case for the 2Hankel approximation when $\boldsymbol{\alpha} \rightarrow \boldsymbol{\alpha}_{\mathbf{0}}$. In the derivation of the 2Hankel approximation, the case when $x_{1}$ and $x_{2}$ are well separated played an important role. For this limiting case, the simple stationary phase approximation (2) is valid, which enabled us to eliminate the unwanted contributions from the stationary phase points $u_{1}$ and $u_{2}$.

In this section, we examine the 2Hankel approximation for the limiting case $\alpha \rightarrow \alpha_{0}$ and ask the question: Do we obtain the transitional Airy approximation (4) in this limit? In eqn (34), (35), (37), (38) and (40) we evidently have to express $g_{1}, f_{1}, f_{1}^{\prime \prime}$, $f_{1}{ }^{\prime \prime \prime}, A_{1}$ and $g_{2}, f_{2}, f_{2}{ }^{\prime \prime}, f_{2}{ }^{\prime \prime \prime}, A_{2}$ in terms of their values at $x_{0}$ rather than at $x_{1}$ and $x_{2}$. Now for $\alpha \rightarrow \alpha_{0}$, we have $x_{1} \approx x_{0} \approx x_{2}$ and to extract the limiting behaviour we approximate $f(\alpha ; x)$ by its 3 -jet at $x_{0}$, as given by eqn (3) or (6).

When the approximation (3) is valid for $f(\alpha ; x)$, the two real stationary phase points, $x_{1} \leq x_{2}$, are the roots of

$$
\left(x-x_{0}\right)^{2}=-2 f_{0}{ }^{\prime} / f_{0}{ }^{\prime \prime \prime}
$$

Since $x_{1}$ and $x_{2}$ are assumed to be real in eqn (42), we must have $f_{0}{ }^{\prime} \leq 0$ (also recall from Section IIA that ${f_{0}}^{\prime \prime \prime}>0$ ). We can write for the two roots

$$
\begin{aligned}
& x_{1}=x_{0}-\sqrt{2\left|f_{0}{ }^{\prime}\right| / f_{0}{ }^{\prime \prime \prime}} \\
& x_{2}=x_{0}+\sqrt{2\left|f_{0}{ }^{\prime}\right| / f_{0}{ }^{\prime \prime \prime}}
\end{aligned}
$$

Now from the cubic approximation (3), we obtain

$$
\begin{gathered}
f^{\prime \prime}(\alpha ; x)=f_{0}{ }^{\prime \prime}\left(x-x_{0}\right) \\
f^{\prime \prime \prime}(\alpha ; x)=f_{0}^{\prime \prime \prime}
\end{gathered}
$$

It follows that for $x=x_{1}$

$$
f_{1}^{\prime \prime}=f_{0}^{\prime \prime \prime}\left(x_{1}-x_{0}\right)=-\sqrt{2\left|f_{0}{ }^{\prime}\right| f_{0}{ }^{\prime \prime \prime}}
$$

and for $x=x_{2}$

$$
f_{2}^{\prime \prime}=f_{0}^{\prime \prime \prime}\left(x_{2}-x_{0}\right)=+\sqrt{2\left|f_{0}{ }^{\prime}\right| f_{0}{ }^{\prime \prime \prime}}
$$

where eqn (43) and (44) have been used. We then find from the definitions (37) and (40) that

$$
A_{1}=A_{2}=\frac{\left(2\left|f_{0}{ }^{\prime}\right|\right)^{3 / 2}}{3\left(f_{0}{ }^{\prime \prime \prime}\right)^{1 / 2}}
$$

To obtain $f_{1}$ and $f_{2}$ we again use eqn (3) together with eqn (43) and (44). The results are

$$
\begin{aligned}
& f_{1}=f_{0}+A_{1} \\
& f_{2}=f_{0}-A_{2}
\end{aligned}
$$

Finally, we make the approximation, $g_{1} \approx g_{0} \approx g_{2}$. Then we can substitute the above results for $g_{1}, f_{1}, f_{1}^{\prime \prime}, f_{2}^{\prime \prime \prime}, A_{1}$ and $g_{2}, f_{2}, f_{2}^{\prime \prime}, f_{2}{ }^{\prime \prime \prime}$, $A_{2}$ into the 2 Hankel approximation, eqn (34), (35), (37), (38) and (40). The last step is to use the identity (14) to replace the two Hankel functions with an Airy function, yielding after simplification

$$
I(\alpha)=2 \pi g_{0} \exp \left(\mathrm{i} f_{0}\right)\left(\frac{2}{f_{0}{ }^{\prime \prime \prime}}\right)^{1 / 3} \operatorname{Ai}\left(-\left|f_{0}^{\prime}\right|\left(\frac{2}{f_{0}{ }^{\prime \prime \prime}}\right)^{1 / 3}\right)
$$

Eqn (45) is the transitional Airy approximation mentioned in Section IIB. Although it has been obtained from the 2 Hankel formula assuming $f_{0}^{\prime} \leq 0$ (two real stationary phase points), it is also valid for $f_{0}^{\prime}>0$, provided $-\left|f_{0}{ }^{\prime}\right|$ is replaced by $f_{0}^{\prime}$ in eqn (45) - see eqn (4).

Thus we have demonstrated that the 2 Hankel approximation contains both limiting cases presented in Section IIB.

\section{Derivation of the 6Hankel approxi- mation for rainbow and glory scattering}

\section{IIIA. Introduction}

Our starting point is the expansion for the scattering amplitude, $f\left(\theta_{\mathrm{R}}\right)$, in a basis set of Legendre polynomials. We write the resulting partial wave series (PWS) in the form

$$
f\left(\theta_{\mathrm{R}}\right)=(2 \mathrm{i} k)^{-1} \sum_{J=0}^{\infty}(2 J+1) \tilde{S}_{J} P_{J}\left(\cos \theta_{\mathrm{R}}\right)
$$

where $k$ is the initial translational wavenumber, $J$ is the total angular momentum quantum number, $\tilde{S}_{J}$ is the $J$ th modified scattering matrix element, and $P_{J}(\bullet)$ is a Legendre polynomial of degree $J$. In order to keep the notation simple, the label, $v_{\mathrm{i}}, j_{\mathrm{i}}, m_{\mathrm{i}} \rightarrow v_{\mathrm{f}}, j_{\mathrm{f}}, m_{\mathrm{f}}$ for the initial and final states, has been omitted from $f\left(\theta_{\mathrm{R}}\right)$ and $\tilde{S}_{J}$, as has the label, $v_{\mathrm{i}}, j_{\mathrm{i}}$, from $k$. Here $v, j, m$ are vibrational, rotational and helicity quantum numbers respectively, with $m_{\mathrm{i}}=m_{\mathrm{f}}=0$ in our case.

We now introduce standard semiclassical approximations into the PWS (46) to convert it into an oscillating integral. ${ }^{3,4}$ Firstly, we transform the PWS into a Poisson series and retain the leading $(m=0)$ term; this assumes all the stationary phase points lie in $(-\pi,+\pi)$, which is the case for our application - see Section IIIB. We have

$$
f\left(\theta_{\mathrm{R}}\right)=(2 \mathrm{i} k)^{-1} \int_{-1 / 2}^{\infty} \mathrm{d} J(2 J+1) \tilde{S}(J) P_{J}\left(\cos \theta_{\mathrm{R}}\right)
$$

In eqn (47), $\tilde{S}(J)$ is the continuation of the set of values, $\left\{\tilde{S}_{J}\right\}$, from integer to real values of $J$ and $P_{J}\left(\cos \theta_{\mathrm{R}}\right)$ is now a Legendre function of the first kind. Secondly, we introduce the Hilb approximation to express the Legendre function in terms of a Bessel function, $J_{0}(\bullet)$, of order zero ${ }^{45,46}$

$$
P_{J}\left(\cos \theta_{\mathrm{R}}\right) \sim \sqrt{\frac{\theta_{\mathrm{R}}}{\sin \theta_{\mathrm{R}}}} J_{0}\left(\left(J+\frac{1}{2}\right) \theta_{\mathrm{R}}\right)
$$


The Hilb approximation has an error, $O\left(J^{-3 / 2}\right)$, which is uniform for $\theta_{\mathrm{R}} \in[0, \pi-\varepsilon]$ with $\varepsilon>0$, We obtain

$$
f\left(\theta_{\mathrm{R}}\right)=\frac{1}{2 \mathrm{i} k} \sqrt{\frac{\theta_{\mathrm{R}}}{\sin \theta_{\mathrm{R}}}} \int_{-1 / 2}^{\infty} \mathrm{d} J(2 J+1) \tilde{S}(J) J_{0}\left(\left(J+\frac{1}{2}\right) \theta_{\mathrm{R}}\right)
$$

Thirdly, we express the Bessel function as a sum of two Hankel functions ${ }^{47}$

$$
J_{0}(x)=\frac{1}{2}\left[H_{0}^{(1)}(x)+H_{0}^{(2)}(x)\right]
$$

We can then write the scattering amplitude as the sum of nearside and farside subamplitudes, ${ }^{3,4,6,19,20}$ which are indicated by superscripts, “(-)” and “(+)”, respectively:

$$
f\left(\theta_{\mathrm{R}}\right)=f^{(-)}\left(\theta_{\mathrm{R}}\right)+f^{(+)}\left(\theta_{\mathrm{R}}\right)
$$

The nearside subamplitude is given by

$$
f^{(-)}\left(\theta_{\mathrm{R}}\right)=\frac{1}{2 \mathrm{i} k} \sqrt{\frac{\theta_{\mathrm{R}}}{\sin \theta_{\mathrm{R}}}} \int_{-1 / 2}^{\infty} \mathrm{d} J\left(J+\frac{1}{2}\right) \tilde{S}(J) H_{0}^{(2)}\left(\left(J+\frac{1}{2}\right) \theta_{\mathrm{R}}\right)
$$

or

$$
\begin{aligned}
f^{(-)}\left(\theta_{\mathrm{R}}\right)= & \frac{1}{2 \mathrm{i} k} \sqrt{\frac{\theta_{\mathrm{R}}}{\sin \theta_{\mathrm{R}}}} \int_{-1 / 2}^{\infty} \mathrm{d} J\left(J+\frac{1}{2}\right)|\tilde{S}(J)| \\
& \times \exp \left\{\mathrm{i}\left[\arg \tilde{S}(J)-\left(J+\frac{1}{2}\right) \theta_{\mathrm{R}}+\pi / 4\right]\right\} \\
& \times\left[\left[\exp \left\{\mathrm{i}\left[\left(J+\frac{1}{2}\right) \theta_{\mathrm{R}}-\pi / 4\right]\right\} H_{0}^{(2)}\left(\left(J+\frac{1}{2}\right) \theta_{\mathrm{R}}\right)\right]\right]
\end{aligned}
$$

The farside subamplitude is given by

$$
f^{(+)}\left(\theta_{\mathrm{R}}\right)=\frac{1}{2 \mathrm{i} k} \sqrt{\frac{\theta_{\mathrm{R}}}{\sin \theta_{\mathrm{R}}}} \int_{-1 / 2}^{\infty} \mathrm{d} J\left(J+\frac{1}{2}\right) \tilde{S}(J) H_{0}^{(1)}\left(\left(J+\frac{1}{2}\right) \theta_{\mathrm{R}}\right)
$$

or

$$
\begin{aligned}
f^{(+)}\left(\theta_{\mathrm{R}}\right)= & \frac{1}{2 \mathrm{i} k} \sqrt{\frac{\theta_{\mathrm{R}}}{\sin \theta_{\mathrm{R}}}} \int_{-1 / 2}^{\infty} \mathrm{d} J\left(J+\frac{1}{2}\right)|\tilde{S}(J)| \\
& \times \exp \left\{\mathrm{i}\left[\arg \tilde{S}(J)+\left(J+\frac{1}{2}\right) \theta_{\mathrm{R}}-\pi / 4\right]\right\} \\
& \times\left[\left[\exp \left\{-\mathrm{i}\left[\left(J+\frac{1}{2}\right) \theta_{\mathrm{R}}-\pi / 4\right]\right\} H_{0}^{(1)}\left(\left(J+\frac{1}{2}\right) \theta_{\mathrm{R}}\right)\right]\right]
\end{aligned}
$$

In writing down eqn (50) and (52), we have followed Miller ${ }^{9}$ and introduced the factors

$$
\exp \left\{ \pm \mathrm{i}\left[\left(J+\frac{1}{2}\right) \theta_{\mathrm{R}}-\pi / 4\right]\right\} \exp \left\{\mp \mathrm{i}\left[\left(J+\frac{1}{2}\right) \theta_{\mathrm{R}}-\pi / 4\right]\right\}=1
$$

This is because the asymptotic eqn (18) and (19) with $\nu=0$ and $\left(J+\frac{1}{2}\right) \theta_{\mathrm{R}} \gg 1$ show that

$$
H_{0}^{(1,2)}\left(\left(J+\frac{1}{2}\right) \theta_{\mathrm{R}}\right) \sim \sqrt{\frac{2}{\pi\left(J+\frac{1}{2}\right) \theta_{\mathrm{R}}}} \exp \left\{ \pm \mathrm{i}\left[\left(J+\frac{1}{2}\right) \theta_{\mathrm{R}}-\pi / 4\right]\right\}
$$

which implies that the expressions in double braces, $\llbracket \bullet \rrbracket$, are relatively slowly varying with respect to $J$.

Remark: As in our previous work, ${ }^{3,4,6}$ we use “₹” to indicate nearside/farside in the semiclassical theory and reserve "N/F" for nearside/farside decompositions in the PWS theory.

The fourth step is the asymptotic evaluation of the oscillatory integrals (50) and (52) using the theory developed in Section II. To do this we must first examine the general properties of $\tilde{S}(J)$ for our application. This is done next.

\section{IIIB. Properties of $\tilde{\boldsymbol{S}}(J)$}

Fig. 2 illustrates the properties of $\tilde{S}(J)$ for the $J$-shifted Eckart model defined in Section IV. In particular, Fig. 2(a) and (b) display $|\tilde{S}(J)|$ and $\arg \tilde{S}(J)$ versus $J$ respectively, whilst Fig. 2(c) is a plot of the quantum deflection function versus $J$, which is defined by

$$
\tilde{\Theta}(J) \equiv \frac{\mathrm{d} \arg \tilde{S}(J)}{\mathrm{d} J}
$$
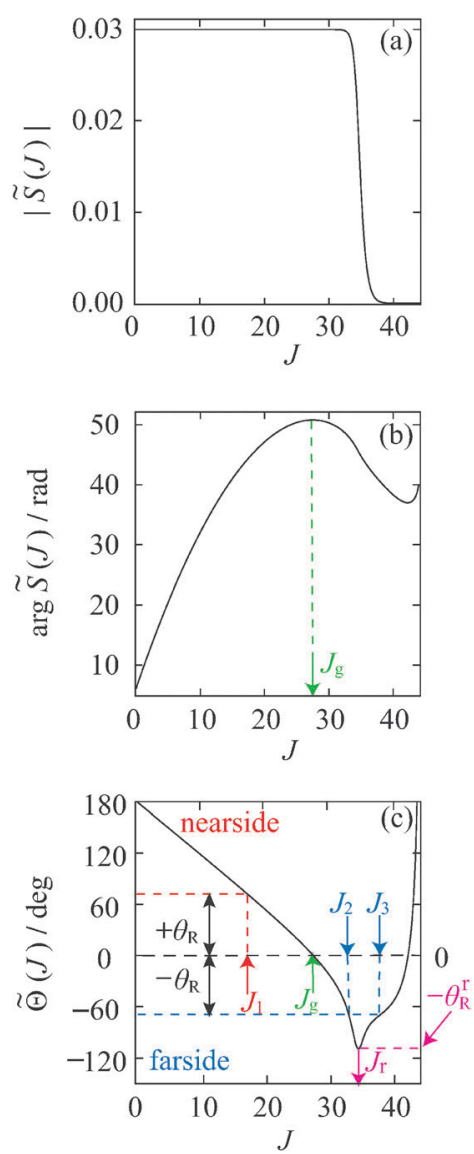

Fig. $2 \mathrm{~S}$ matrix data for the J-shifted Eckart parameterization. The values of the parameters are given in Section IVB. (a) $|\tilde{S}(J)|$ versus J. (b) arg $\tilde{S}(J) /$ rad versus $J$. The maximum of the $\arg \tilde{S}(J) / r a d$ curve defines the glory angular momentum variable, $J_{g}$, which is indicated by a green dashed line and arrow. (c) $\tilde{\Theta}(J) / d e g$ versus $\mathrm{J}$. The red dashed lines and arrow indicate $+\theta_{R}$ and $J_{1}=J_{1}\left(\theta_{R}\right)$ for the nearside scattering. The blue dashed lines and arrows indicate $-\theta_{R}$, as well as $J_{2}=J_{2}\left(\theta_{R}\right)$ and $J_{3}=J_{3}\left(\theta_{R}\right)$ for the farside scattering. Also shown is the rainbow angular momentum variable, $J_{r}$, which is located at the minimum of the $\tilde{\Theta}(J) /$ deg curve, where $\tilde{\Theta}\left(J_{r}\right)=-\theta_{R}^{r}$ (pink arrow and dashed line respectively), together with $J_{g}$, which satisfies the equation $\tilde{\Theta}\left(J_{g}\right)=0$ (green arrow). 
For eqn (50) and (52), the stationary phase points are the roots of

$$
\tilde{\Theta}(J) \mp \theta_{\mathrm{R}}=0
$$

where "-" corresponds to nearside scattering and "+" to farside scattering. We see there can be a contribution from both the nearside and farside scattering at a given value of $\theta_{\mathrm{R}}$; we consider the nearside and farside cases separately.

\section{IIIC. Nearside scattering}

Fig. 2(c) shows there is a single real root to $\tilde{\Theta}(J)=+\theta_{\mathrm{R}}$, which we denote by $J_{1} \equiv J_{1}\left(\theta_{\mathrm{R}}\right)$. The asymptotic theory developed in Section IIC.3 applies, in particular eqn (29).

We make the following identifications between eqn (1) and (50)

$$
\begin{gathered}
x=J, \quad x_{1}=J_{1}, \quad \alpha=\theta_{\mathrm{R}} \\
g(x)=\left(J+\frac{1}{2}\right)|\tilde{S}(J)| \\
\times\left[\left[\exp \left\{\mathrm{i}\left[\left(J+\frac{1}{2}\right) \theta_{\mathrm{R}}-\pi / 4\right]\right\} H_{0}^{(2)}\left(\left(J+\frac{1}{2}\right) \theta_{\mathrm{R}}\right)\right]\right] \\
f(\alpha ; x)=\arg \tilde{S}(J)-\left(J+\frac{1}{2}\right) \theta_{\mathrm{R}}+\pi / 4
\end{gathered}
$$

so that

$$
\begin{gathered}
f^{\prime}(\alpha ; x)=\tilde{\Theta}(J)-\theta_{\mathrm{R}} \\
f^{\prime \prime}(\alpha ; x)=\tilde{\Theta}^{\prime}(J) \\
f^{\prime \prime \prime}(\alpha ; x)=\tilde{\Theta}^{\prime \prime}(J)
\end{gathered}
$$

Note that the phase has a linear dependence on $\theta_{\mathrm{R}}$, namely, $-\left(J+\frac{1}{2}\right) \theta_{\mathrm{R}}$. Then from eqn (28), (29) and (50), we can write for the nearside subamplitude to the 6Hankel approximation

$$
\begin{aligned}
f_{6 \mathrm{H}}^{(-)}\left(1 \mid \theta_{\mathrm{R}}\right)= & \frac{1}{2 \mathrm{i} k} \sqrt{\frac{\theta_{\mathrm{R}}}{\sin \theta_{\mathrm{R}}}} \frac{\pi}{3^{1 / 6}} \\
& \times\left[\frac{B_{1}\left(\theta_{\mathrm{R}}\right)}{\left|\tilde{\Theta}^{\prime \prime}\left(J_{1}\left(\theta_{\mathrm{R}}\right)\right)\right|}\right]^{1 / 3}\left[J_{1}\left(\theta_{\mathrm{R}}\right)+\frac{1}{2}\right]\left|\tilde{S}\left(J_{1}\left(\theta_{\mathrm{R}}\right)\right)\right| \\
& \times \exp \left\{\mathrm{i}\left[\arg \tilde{S}\left(J_{1}\left(\theta_{\mathrm{R}}\right)\right)-B_{1}\left(\theta_{\mathrm{R}}\right)+\pi / 6\right]\right\} \\
& \times H_{0}^{(2)}\left(\left[J_{1}\left(\theta_{\mathrm{R}}\right)+\frac{1}{2}\right] \theta_{\mathrm{R}}\right) H_{1 / 3}^{(1)}\left(B_{1}\left(\theta_{\mathrm{R}}\right)\right)
\end{aligned}
$$

with

$$
B_{1}\left(\theta_{\mathrm{R}}\right)=\frac{\left|\tilde{\Theta}^{\prime}\left(J_{1}\left(\theta_{\mathrm{R}}\right)\right)\right|^{3}}{3\left[\tilde{\Theta}^{\prime \prime}\left(J_{1}\left(\theta_{\mathrm{R}}\right)\right)\right]^{2}}
$$

\section{IIID. Farside scattering}

Perusal of Fig. 2(c) shows there are two real roots to $\tilde{\Theta}(J)=-\theta_{\mathrm{R}}$, which we denote $J_{2} \equiv J_{2}\left(\theta_{\mathrm{R}}\right)$ and $J_{3} \equiv J_{3}\left(\theta_{\mathrm{R}}\right)$ with $J_{2} \leq J_{3}$. At the rainbow angle, $\theta_{\mathrm{R}}=\theta_{\mathrm{R}}^{\mathrm{r}}, J_{2}$ and $J_{3}$ coalesce to $J_{\mathrm{r}}$, the value of the rainbow angular momentum variable; we then have, $\tilde{\Theta}\left(J_{\mathrm{r}}\right)=-\theta_{\mathrm{R}}^{\mathrm{r}}$. For the farside scattering, the asymptotic theory developed in Section IIC.4 applies and in particular we can use the 2Hankel approximation.

We make the following identifications between eqn (1) and (52)

$$
x=J, \quad x_{1}=J_{2}, \quad x_{2}=J_{3}, \quad \alpha=\theta_{\mathrm{R}}
$$

$g(x)=\left(J+\frac{1}{2}\right)|\tilde{S}(J)|$

$$
\times\left[\left[\exp \left\{-\mathrm{i}\left[\left(J+\frac{1}{2}\right) \theta_{\mathrm{R}}-\pi / 4\right]\right\} H_{0}^{(1)}\left(\left(J+\frac{1}{2}\right) \theta_{\mathrm{R}}\right)\right]\right]
$$

$$
f(\alpha ; x)=\arg \tilde{S}(J)+\left(J+\frac{1}{2}\right) \theta_{\mathrm{R}}-\pi / 4
$$

so that

$$
\begin{gathered}
f^{\prime}(\alpha ; x)=\tilde{\Theta}(J)+\theta_{\mathrm{R}} \\
f^{\prime \prime}(\alpha ; x)=\tilde{\Theta}^{\prime}(J) \\
f^{\prime \prime \prime}(\alpha ; x)=\tilde{\Theta}^{\prime \prime}(J)
\end{gathered}
$$

Note that the phase has a linear dependence on $\theta_{\mathrm{R}}$, namely, $+\left(J+\frac{1}{2}\right) \theta_{\mathrm{R}}$. Then from eqn (34), (35), (37), (38), (40) and (52) we have for the two farside subamplitudes which contribute to the 6 Hankel approximation

$$
\begin{aligned}
f_{6 \mathrm{H}}^{(+)}\left(2 \mid \theta_{\mathrm{R}}\right)= & \frac{1}{2 \mathrm{i} k} \sqrt{\frac{\theta_{\mathrm{R}}}{\sin \theta_{\mathrm{R}}}} \frac{\pi}{3^{1 / 6}} \\
& \times\left[\frac{B_{2}\left(\theta_{\mathrm{R}}\right)}{\left|\tilde{\Theta}^{\prime \prime}\left(J_{2}\left(\theta_{\mathrm{R}}\right)\right)\right|}\right]^{1 / 3}\left[J_{2}\left(\theta_{\mathrm{R}}\right)+\frac{1}{2}\right]\left|\tilde{S}\left(J_{2}\left(\theta_{\mathrm{R}}\right)\right)\right| \\
& \times \exp \left\{\mathrm{i}\left[\arg \tilde{S}\left(J_{2}\left(\theta_{\mathrm{R}}\right)\right)-B_{2}\left(\theta_{\mathrm{R}}\right)+\pi / 6\right]\right\} \\
& \times H_{0}^{(1)}\left(\left[J_{2}\left(\theta_{\mathrm{R}}\right)+\frac{1}{2}\right] \theta_{\mathrm{R}}\right) H_{1 / 3}^{(1)}\left(B_{2}\left(\theta_{\mathrm{R}}\right)\right)
\end{aligned}
$$

with

$$
B_{2}\left(\theta_{\mathrm{R}}\right)=\frac{\left|\tilde{\Theta}^{\prime}\left(J_{2}\left(\theta_{\mathrm{R}}\right)\right)\right|^{3}}{3\left[\tilde{\Theta}^{\prime \prime}\left(J_{2}\left(\theta_{\mathrm{R}}\right)\right)\right]^{2}}
$$

and

$$
\begin{aligned}
f_{6 \mathrm{H}}^{(+)}\left(3 \mid \theta_{\mathrm{R}}\right)= & \frac{1}{2 \mathrm{i} k} \sqrt{\frac{\theta_{\mathrm{R}}}{\sin \theta_{\mathrm{R}}}} \frac{\pi}{3^{1 / 6}} \\
& \times\left[\frac{B_{3}\left(\theta_{\mathrm{R}}\right)}{\left|\tilde{\Theta}^{\prime \prime}\left(J_{3}\left(\theta_{\mathrm{R}}\right)\right)\right|}\right]^{1 / 3}\left[J_{3}\left(\theta_{\mathrm{R}}\right)+\frac{1}{2}\right]\left|\tilde{S}\left(J_{3}\left(\theta_{\mathrm{R}}\right)\right)\right| \\
& \times \exp \left\{\mathrm{i}\left[\arg \tilde{S}\left(J_{3}\left(\theta_{\mathrm{R}}\right)\right)+B_{3}\left(\theta_{\mathrm{R}}\right)-\pi / 6\right]\right\} \\
& \times H_{0}^{(1)}\left(\left[J_{3}\left(\theta_{\mathrm{R}}\right)+\frac{1}{2}\right] \theta_{\mathrm{R}}\right) H_{1 / 3}^{(2)}\left(B_{3}\left(\theta_{\mathrm{R}}\right)\right)
\end{aligned}
$$


with

$$
B_{3}\left(\theta_{\mathrm{R}}\right)=\frac{\tilde{\Theta}^{\prime}\left(J_{3}\left(\theta_{\mathrm{R}}\right)\right)^{3}}{3\left[\tilde{\Theta}^{\prime \prime}\left(J_{3}\left(\theta_{\mathrm{R}}\right)\right)\right]^{2}}
$$

\section{IIIE. 6Hankel approximation}

The 6Hankel approximation for the full scattering amplitude is then obtained by summing the subamplitudes (53), (55) and (57)

$$
f_{6 \mathrm{H}}\left(\theta_{\mathrm{R}}\right)=f_{6 \mathrm{H}}^{(-)}\left(1 \mid \theta_{\mathrm{R}}\right)+f_{6 \mathrm{H}}^{(+)}\left(2 \mid \theta_{\mathrm{R}}\right)+f_{6 \mathrm{H}}^{(+)}\left(3 \mid \theta_{\mathrm{R}}\right)
$$

The corresponding DCS is

$$
\sigma_{6 \mathrm{H}}\left(\theta_{\mathrm{R}}\right)=\left|f_{6 \mathrm{H}}\left(\theta_{\mathrm{R}}\right)\right|^{2}
$$

Eqn (59) is the 6Hankel approximation that was written down without proof in our recent paper with Zhang. ${ }^{6} \mathrm{We}$ see that it contains six Hankel functions, three of order zero, $H_{0}^{(1,2)}(\bullet)$, and three of order $1 / 3, H_{1 / 3}^{(1,2)}(\bullet)$.

Note that the farside subamplitudes (55) and (57) are only valid on the bright side of the rainbow angle, where the two roots of $\tilde{\Theta}(J)=-\theta_{\mathrm{R}}$ are real. Thus in our calculations in Section $\mathrm{V}$, we will only apply the full 6 Hankel approximation (59) for $0<\theta_{\mathrm{R}}<\theta_{\mathrm{R}}^{\mathrm{r}}$.

When the arguments of the Hankel functions in eqn (53), (55) and (57) are large, we can substitute the asymptotic results (18) and (19) with $\nu=0$ or $1 / 3$. We then obtain the following expressions for the subamplitudes, which constitute the primitive semiclassical approximation (PSA):

$$
\begin{gathered}
f_{6 \mathrm{H}}^{(-)}\left(1 \mid \theta_{\mathrm{R}}\right) \sim f_{\mathrm{PSA}}^{(-)}\left(1 \mid \theta_{\mathrm{R}}\right)=-\mathrm{i} \sqrt{\sigma_{1}^{(-)}\left(\theta_{\mathrm{R}}\right)} \exp \left[\mathrm{i} \beta_{1}^{(-)}\left(\theta_{\mathrm{R}}\right)\right] \\
f_{6 \mathrm{H}}^{(+)}\left(2 \mid \theta_{\mathrm{R}}\right) \sim f_{\mathrm{PSA}}^{(+)}\left(2 \mid \theta_{\mathrm{R}}\right)=-\sqrt{\sigma_{2}^{(+)}\left(\theta_{\mathrm{R}}\right)} \exp \left[\mathrm{i} \beta_{2}^{(+)}\left(\theta_{\mathrm{R}}\right)\right] \\
f_{6 \mathrm{H}}^{(+)}\left(3 \mid \theta_{\mathrm{R}}\right) \sim f_{\mathrm{PSA}}^{(+)}\left(3 \mid \theta_{\mathrm{R}}\right)=-\mathrm{i} \sqrt{\sigma_{3}^{(+)}\left(\theta_{\mathrm{R}}\right)} \exp \left[\mathrm{i} \beta_{3}^{(+)}\left(\theta_{\mathrm{R}}\right)\right]
\end{gathered}
$$

where the "classical-like" DCSs are given by

$$
\begin{gathered}
\sigma_{1}^{(-)}\left(\theta_{\mathrm{R}}\right)=\frac{\left[J_{1}\left(\theta_{\mathrm{R}}\right)+\frac{1}{2}\right]\left|\tilde{S}\left(J_{1}\left(\theta_{\mathrm{R}}\right)\right)\right|^{2}}{k^{2} \sin \theta_{\mathrm{R}}\left|\tilde{\Theta}^{\prime}\left(J_{1}\left(\theta_{\mathrm{R}}\right)\right)\right|} \\
\sigma_{2}^{(+)}\left(\theta_{\mathrm{R}}\right)=\frac{\left[J_{2}\left(\theta_{\mathrm{R}}\right)+\frac{1}{2}\right]\left|\tilde{S}\left(J_{2}\left(\theta_{\mathrm{R}}\right)\right)\right|^{2}}{k^{2} \sin \theta_{\mathrm{R}}\left|\tilde{\Theta}^{\prime}\left(J_{2}\left(\theta_{\mathrm{R}}\right)\right)\right|} \\
\sigma_{3}^{(+)}\left(\theta_{\mathrm{R}}\right)=\frac{\left[J_{3}\left(\theta_{\mathrm{R}}\right)+\frac{1}{2}\right]\left|\tilde{S}\left(J_{3}\left(\theta_{\mathrm{R}}\right)\right)\right|^{2}}{k^{2} \sin \theta_{\mathrm{R}} \tilde{\Theta}^{\prime}\left(J_{3}\left(\theta_{\mathrm{R}}\right)\right)}
\end{gathered}
$$

and the phases are

$$
\begin{aligned}
& \beta_{1}^{(-)}\left(\theta_{\mathrm{R}}\right)=\arg \tilde{S}\left(J_{1}\left(\theta_{\mathrm{R}}\right)\right)-\left[J_{1}\left(\theta_{\mathrm{R}}\right)+\frac{1}{2}\right] \theta_{\mathrm{R}} \\
& \beta_{2}^{(+)}\left(\theta_{\mathrm{R}}\right)=\arg \tilde{S}\left(J_{2}\left(\theta_{\mathrm{R}}\right)\right)+\left[J_{2}\left(\theta_{\mathrm{R}}\right)+\frac{1}{2}\right] \theta_{\mathrm{R}} \\
& \beta_{3}^{(+)}\left(\theta_{\mathrm{R}}\right)=\arg \tilde{S}\left(J_{3}\left(\theta_{\mathrm{R}}\right)\right)+\left[J_{3}\left(\theta_{\mathrm{R}}\right)+\frac{1}{2}\right] \theta_{\mathrm{R}}
\end{aligned}
$$

In a systematic semiclassical (SC) notation, ${ }^{4,6}$ eqn (61)-(63) are written SC/N/PSA. We call the sum of the two farside subamplitudes, SC/F/PSA.

\section{IIIF. The limit $\boldsymbol{\theta}_{\mathrm{R}} \rightarrow \boldsymbol{\theta}_{\mathrm{R}}^{\mathrm{r}}$ : farside rainbow scattering}

When $\theta_{\mathrm{R}} \rightarrow \theta_{\mathrm{R}}^{\mathrm{r}}, f_{6 \mathrm{H}}^{(+)}\left(2 \mid \theta_{\mathrm{R}}\right)+f_{6 \mathrm{H}}^{(+)}\left(3 \mid \theta_{\mathrm{R}}\right)$ becomes numerically unstable because of subtractive cancellation. This arises because $\tilde{\Theta}^{\prime}\left(J_{2}\left(\theta_{\mathrm{R}}\right)\right) \rightarrow 0$ and $\tilde{\Theta}^{\prime}\left(J_{3}\left(\theta_{\mathrm{R}}\right)\right) \rightarrow 0$ as the rainbow angle is approached, and the asymptotic limits (41) apply to the $H_{1 / 3}^{(1)}$ $\left(B_{i}\left(\theta_{\mathrm{R}}\right)\right)$ with $i=1$ and 2 . We examined this limit in Section IIC.5, and showed that the 2Hankel approximation contains the transitional Airy approximation (45) [or eqn (4)] when $\alpha \rightarrow \alpha_{0}$. We can apply this result to the farside scattering, as given by eqn (55) and (57). Making the identifications

$$
x=J, \quad x_{0}=J_{\mathrm{r}}, \quad \alpha=\theta_{\mathrm{R}}, \quad \alpha_{0}=\theta_{\mathrm{R}}^{\mathrm{r}}
$$

we can write for the farside subamplitude.

$$
\begin{aligned}
f_{\text {tAiry }}^{(+)}\left(\theta_{\mathrm{R}}\right)= & \frac{1}{k} \sqrt{\frac{2 \pi\left(J_{\mathrm{r}}+\frac{1}{2}\right)}{\sin \theta_{\mathrm{R}}}} \frac{\left|\tilde{S}\left(J_{\mathrm{r}}\right)\right|}{q_{\mathrm{r}}{ }^{1 / 3}} \\
& \times \exp \left\{\mathrm{i}\left[\arg \tilde{S}\left(J_{\mathrm{r}}\right)+\left(J_{\mathrm{r}}+\frac{1}{2}\right) \theta_{\mathrm{R}}-3 \pi / 4\right]\right\} \\
& \times \operatorname{Ai}\left(\frac{\theta_{\mathrm{R}}-\theta_{\mathrm{R}}^{\mathrm{r}}}{q_{\mathrm{r}}{ }^{1 / 3}}\right)
\end{aligned}
$$

where

$$
q_{\mathrm{r}}=\left.\frac{1}{2} \frac{\mathrm{d}^{2} \tilde{\Theta}(J)}{\mathrm{d} J^{2}}\right|_{J=J_{\mathrm{r}}}=\left.\frac{1}{2} \frac{\mathrm{d}^{3} \arg \tilde{S}(J)}{\mathrm{d} J^{3}}\right|_{J=J_{\mathrm{r}}}
$$

The approximation (64) is equivalent to replacing $\tilde{\Theta}(J)$ by its 2 -jet at $J_{\mathrm{r}}$, namely

$$
j_{J_{\mathrm{r}}}^{2} \tilde{\Theta}(J)=-\theta_{\mathrm{R}}^{\mathrm{r}}+q_{\mathrm{r}}\left(J-J_{\mathrm{r}}\right)^{2}
$$

Eqn (64) has the advantage that it can be used on both the bright side, $\theta_{\mathrm{R}}<\theta_{\mathrm{R}}^{\mathrm{r}}$, and the dark side, $\theta_{\mathrm{R}}>\theta_{\mathrm{R}}^{\mathrm{r}}$, of the rainbow, as well as at $\theta_{\mathrm{R}}=\theta_{\mathrm{R}}^{\mathrm{r}}$.

Remarks:

- In a systematic semiclassical notation, ${ }^{4,6}$ eqn (64) is written as $\mathrm{SC} / \mathrm{F} /$ tAiry, or tAiry for short.

- When the tAiry subamplitude (64) is used to calculate the DCS, we must also include the contribution from the nearside scattering. We use the SC/N/PSA subamplitude of eqn (61)-(63) for this purpose.

- It is known that the tAiry subamplitude is a special case of the more general uniform Airy approximation, as derived in ref. 4 and 6. In a systematic notation, ${ }^{4,6}$ it is described as SC/F/uAiry, or uAiry for short.

\section{IIIG. The limit $\theta_{\mathrm{R}} \rightarrow \mathbf{0}$ : glory scattering}

Finally we must examine the glory limit, $\theta_{\mathrm{R}} \rightarrow 0$, for $f_{6 \mathrm{H}}^{(-)}\left(1 \mid \theta_{\mathrm{R}}\right)+$ $f_{6 \mathrm{H}}^{(+)}\left(2 \mid \theta_{\mathrm{R}}\right)$. In eqn (53) and (55), we note the following limits as 
$\theta_{\mathrm{R}} \rightarrow 0$, for $i=1$ or 2 ;

$$
\begin{gathered}
\sqrt{\frac{\theta_{\mathrm{R}}}{\sin \theta_{\mathrm{R}}}} \rightarrow 1 \\
J_{i}\left(\theta_{\mathrm{R}}\right) \rightarrow J_{i}(0) \equiv J_{\mathrm{g}}, \\
\tilde{\Theta}^{\prime}\left(J_{i}\left(\theta_{\mathrm{R}}\right)\right) \rightarrow \tilde{\Theta}^{\prime}\left(J_{i}(0)\right)=\tilde{\Theta}^{\prime}\left(J_{\mathrm{g}}\right) \equiv \tilde{\Theta}_{\mathrm{g}}{ }^{\prime} \\
\tilde{\Theta}^{\prime \prime}\left(J_{i}\left(\theta_{\mathrm{R}}\right)\right) \rightarrow \tilde{\Theta}^{\prime \prime}\left(J_{i}(0)\right)=\tilde{\Theta}^{\prime \prime}\left(J_{\mathrm{g}}\right) \equiv \tilde{\Theta}_{\mathrm{g}}^{\prime \prime} \\
B_{i}\left(\theta_{\mathrm{R}}\right) \rightarrow B_{i}(0) \equiv B_{\mathrm{g}} \\
\tilde{S}\left(J_{i}\left(\theta_{\mathrm{R}}\right)\right) \rightarrow \tilde{S}\left(J_{i}(0)\right)=\tilde{S}\left(J_{\mathrm{g}}\right) \equiv \tilde{S}_{\mathrm{g}} \\
H_{1 / 3}^{(1)}\left(B_{i}\left(\theta_{\mathrm{R}}\right)\right) \rightarrow H_{1 / 3}^{(1)}\left(B_{i}(0)\right) \equiv H_{1 / 3}^{(1)}\left(B_{\mathrm{g}}\right)
\end{gathered}
$$

We can use the above limits and the identity (48) to deduce that in the limit $\theta_{\mathrm{R}} \rightarrow 0$

$$
H_{0}^{(2)}\left(\left[J_{1}\left(\theta_{\mathrm{R}}\right)+\frac{1}{2}\right] \theta_{\mathrm{R}}\right)+H_{0}^{(1)}\left(\left[J_{2}\left(\theta_{\mathrm{R}}\right)+\frac{1}{2}\right] \theta_{\mathrm{R}}\right) \rightarrow 2 J_{0}(0)=2
$$

We then obtain

$$
\begin{aligned}
& f_{6 \mathrm{H}}^{(-)}\left(1 \mid \theta_{\mathrm{R}}\right)+f_{6 \mathrm{H}}^{(+)}\left(2 \mid \theta_{\mathrm{R}}\right) \\
& \quad=\frac{1}{\mathrm{i} k} \frac{\pi}{3^{1 / 6}}\left(\frac{B_{\mathrm{g}}}{\left|\tilde{\Theta}_{\mathrm{g}}{ }^{\prime \prime}\right|}\right)^{1 / 3}\left(J_{\mathrm{g}}+\frac{1}{2}\right) \tilde{S}_{\mathrm{g}} \exp \left[-\mathrm{i}\left(B_{\mathrm{g}}-\frac{\pi}{6}\right)\right] H_{1 / 3}^{(1)}\left(B_{\mathrm{g}}\right)
\end{aligned}
$$

where

$$
B_{\mathrm{g}}=\frac{\left|\tilde{\Theta}^{\prime}\left(J_{\mathrm{g}}\right)\right|^{3}}{3\left[\tilde{\Theta}^{\prime \prime}\left(J_{\mathrm{g}}\right)\right]^{2}}
$$

Inspection of Fig. 2(c) shows that we expect $\tilde{\Theta}^{\prime \prime}\left(J_{\mathrm{g}}\right)$ to be very small in magnitude; hence from eqn (66), we conclude that $B_{\mathrm{g}} \gg 1$, and so can use the asymptotic approximation (18) with $\nu=1 / 3$ in eqn (65). We obtain for the limit $\theta_{\mathrm{R}} \rightarrow 0$

$$
f_{6 \mathrm{H}}^{(-)}\left(1 \mid \theta_{\mathrm{R}}\right)+f_{6 \mathrm{H}}^{(+)}\left(2 \mid \theta_{\mathrm{R}}\right) \rightarrow-\frac{\exp (\mathrm{i} \pi / 4)}{k} \sqrt{\frac{2 \pi}{\left|\tilde{\Theta}_{\mathrm{g}}^{\prime}\right|}}\left(J_{\mathrm{g}}+\frac{1}{2}\right) \tilde{S}_{\mathrm{g}}
$$

which is recognized as the semiclassical transitional approximation (STA) at $\theta_{\mathrm{R}}=0$, as given by eqn (14) of ref. 3 or eqn (29) of ref. 7 .

Remarks:

- The contribution from $f_{6 \mathrm{H}}^{(+)}\left(3 \mid \theta_{\mathrm{R}}\right)$ to the scattering at $\theta_{\mathrm{R}} \approx 0$ is very small in our application in Section $\mathrm{V}$. It has been neglected in the above derivation.

- $f_{6 \mathrm{H}}^{(-)}\left(1 \mid \theta_{\mathrm{R}}\right)$ and $f_{6 \mathrm{H}}^{(+)}\left(2 \mid \theta_{\mathrm{R}}\right)$ become large in magnitude as $\theta_{\mathrm{R}} \rightarrow 0$, but their sum $f_{6 \mathrm{H}}^{(-)}\left(1 \mid \theta_{\mathrm{R}}\right)+f_{6 \mathrm{H}}^{(+)}\left(2 \mid \theta_{\mathrm{R}}\right)$ is much smaller. This implies the 6 Hankel approximation becomes numerically unstable as $\theta_{\mathrm{R}} \rightarrow 0$.

- The STA is a special case of the uniform semiclassical approximation (USA) for forward glory scattering which was derived and discussed in ref. 3 and 7. However, in this paper we will use the more explicit name, uniform Bessel approximation, and use the abbreviation, uBessel.

\section{IIIH. Discussion}

We note the following:

- The 6Hankel approximation (59) is generic. This means it is applicable to numerous chemical reactions at numerous collision energies (in principle, an infinite number of cases) which have $\mathrm{S}$ matrix elements that are analogous to those in Fig. 2.

- The 6Hankel approximation (59) is uniform for both rainbow and glory scattering, with the advantage that the contributions from all three roots $J_{1}, J_{2}, J_{3}$ appear separately. In contrast, in the standard semiclassical approach, it is necessary to apply two different theories: the uniform rainbow theory (SC/F/uAiry or uAiry), ${ }^{4,6}$ which involves the pair of roots, $J_{2}$ and $J_{3}$; and the uniform glory theory (uBessel), ${ }^{3,7}$ which involves the pair $J_{1}$ and $J_{2}$.

- The 6Hankel approximation (59) has the disadvantage that it involves third derivatives of $\arg \tilde{S}(J)$; these can be difficult to calculate accurately for numerical input data. Moreover in practical applications, these third derivatives can change sign. In contrast, the uAiry and uBessel theories involve just the second derivatives.

- The 6Hankel approximation (59) has the disadvantage that it becomes numerically unstable for $\theta_{\mathrm{R}} \rightarrow 0$ and for $\theta_{\mathrm{R}} \rightarrow \theta_{\mathrm{R}}^{\mathrm{r}}$, in particular for numerical $\left\{\tilde{S}_{J}\right\}$ that possess errors. These problems can be overcome by using the STA for glory scattering and the tAiry approximation for rainbow scattering.

- The 6 Hankel formula for the DCS is a generalization to reactive scattering of a result given by Miller for elastic collisions. In particular, if we set in eqn (53)-(60), $|\tilde{S}(J)| \rightarrow 1$ and arg $\tilde{S}(J) \rightarrow 2 \delta(J)$, where $\delta(J)$ is the elastic phase shift, then, after considerable algebraic manipulations, we obtain for $\sigma_{6 \mathrm{H}}\left(\theta_{\mathrm{R}}\right)$ the result quoted by Miller [eqn (6) of ref. 9]. One difference is that Miller assumes the Wentzel-Kramers-Brillouin approximation for $\delta(J)$, whereas our derivation shows that this assumption is not necessary.

\section{J-shifted Eckart parameterization and the values of its parameters}

This section defines the $J$-shifted Eckart parameterization for $\tilde{S}(J)$ in Section IVA, whilst Section IVB gives the values of its parameters that are used in our DCS computations in Section V.

\section{IVA. J-shifted Eckart parameterization}

Sokolovski introduced the $J$-shifted Eckart parameterization for $\tilde{S}(J)$ in order to model the DCS for the state-selected $\mathrm{H}+\mathrm{D}_{2} \rightarrow$ $\mathrm{HD}+\mathrm{D}$ reaction; in particular to mimic a resonance near the reaction threshold. ${ }^{10,48}$ It has subsequently been found to be very useful for understanding the dynamics of chemical reactions. ${ }^{11,48,49}$ The $J$-shifted Eckart parameterization is based on the exact quantum solution for a particle of reduced mass $\mu$ transmitted by a symmetric Eckart potential, $W_{0} / \cosh ^{2}\left(s / s_{0}\right)$, where $s$ is a reaction coordinate, $s_{0}$ is a reference distance and 
$W_{0}$ is the height of the energy barrier. The following formulae define this parameterization:

$$
\left.\begin{array}{rlrl}
\tilde{S}(J) & =\tilde{S}(J) \exp [\mathrm{i} \tilde{\phi}(J)], & J=0,1,2, \ldots, J_{\max } \\
& =0 & J=J_{\max }+1, J_{\max }+2, \ldots
\end{array}\right\}
$$

where

$$
\tilde{\phi}(J)=a J^{2}+b J
$$

is a quadratic phase, with real parameters, $a$ and $b$. Also

$$
\tilde{s}(J)=N \frac{\Gamma\left(-\mathrm{i} K(J)+\mathrm{i} Q+\frac{1}{2}\right) \Gamma\left(-\mathrm{i} K(J)-\mathrm{i} Q+\frac{1}{2}\right)}{\Gamma(-\mathrm{i} K(J)) \Gamma(1-\mathrm{i} K(J))}
$$

In addition

- $N=$ scaling factor (dimensionless).

- $Q=\sqrt{W_{0} / \varepsilon-1 / 4}$ (dimensionless because of the following definition).

- $\varepsilon=\hbar^{2} /\left(2 \mu s_{0}^{2}\right)$ (dimensions of energy).

- $K(J)=\sqrt{[E-B J(J+1)] / \varepsilon}$ (dimensionless). In this equation, $E$ is the total energy and $B$ is the rotational constant for the triatomic complex. The term $B J(J+1)$ represents the $J$-shifted approximation, because it introduces a $J$ dependence into the mathematically one-dimensional Eckart barrier.

- $K(J)$ has two branch points at real values of $J$, which are the roots of $E-B J(J+1)=0$. The larger root is located at $J_{\mathrm{c}}=\sqrt{E / B+1 / 4}-1 / 2$. The integer $J_{\max }$ is then defined by $J_{\max }=\operatorname{Floor}\left(J_{\mathrm{c}}\right)$. It follows that $K\left(J_{\max }\right)$ is real and $K\left(J_{\max }+1\right)$ is purely imaginary. In our application in Section $\mathrm{V}$, we have $J_{\max } \gg 1$.

\section{IVB. Parameter values for the $J$-shifted Eckart} parameterization

We used the following values for the parameters

$$
\left.\begin{array}{lll}
a=-0.052, & b=\pi, & N=0.03 \\
E / \varepsilon=226.54, & B / \varepsilon=0.11247, & W_{0} / \varepsilon=90
\end{array}\right\}
$$

which results in $J_{\max }=44$. Plots of $|\tilde{S}(J)|$, arg $\tilde{S}(J)$ and $\tilde{\Theta}(J)$ versus $J$ respectively have already been shown and discussed in Fig. 2(a), (b) and (c) respectively. In Section $\mathrm{V}$, we will require the position of the leading Regge pole $(n=0)$ in the first quadrant of the complex angular momentum plane for the $J$-shifted Eckart potential with the parameter values (67). It is obtained from the poles of $\Gamma(-\mathrm{i} K(J)+\mathrm{i} Q+1 / 2)$ and is given by $J_{0}=34.4+1.21 \mathrm{i}$, which corresponds to a life-angle of $1 /\left(2 \mathrm{Im} J_{0}\right)=$ $0.415 \mathrm{rad}=23.8^{\circ}$.

Remark: the parameter values (67) are based on the "standard values" employed for the $\mathrm{H}+\mathrm{D}_{2}$ reaction, ${ }^{10,11}$ which have $B / \varepsilon=$ 0.12247 and $W_{0} / \varepsilon=150$. However, the standard values result in $\theta_{\mathrm{R}}^{\mathrm{r}}=24.0^{\circ}$ at $J_{\mathrm{r}}=25.2$, which only allows a test of the 6 Hankel approximation over a relatively small range of angles. Changing $B / \varepsilon$ to 0.11247 and $W_{0} / \varepsilon$ to 90 gives $\theta_{\mathrm{R}}^{\mathrm{r}}=109.2^{\circ}$ at $J_{\mathrm{r}}=34.5$, which permits the semiclassical approximations to be tested over a wider range of $\theta_{\mathrm{R}}$.

\section{Results for the J-shifted Eckart parameterization}

This section presents our results for the angular scattering in Section VA, whilst Section VB reports a nearside-farside (NF) analysis of the (dimensionless) DCS, as well as a NF analysis for the local angular momentum (LAM). All the semiclassical DCSs are defined as the square modulus of the corresponding scattering amplitude.

\section{VA. Dimensionless differential cross sections}

Fig. 3 plots, on a linear scale, the dimensionless differential cross section (dDCS), $k^{2} \sigma\left(\theta_{\mathrm{R}}\right)$, versus $\theta_{\mathrm{R}}$ for the PWS, where it is compared with the uAiry + SC/N/PSA, uBessel and 6Hankel approximations for $\theta_{\mathrm{R}}<\theta_{\mathrm{R}}^{\mathrm{r}}$. When $\theta_{\mathrm{R}} \geq \theta_{\mathrm{R}}^{\mathrm{r}}$, the dDCS for the tAiry + SC/N/PSA approximation is drawn. For consistency with ref. 4, the tAiry subamplitude includes the first correction term to eqn (64), even though it makes only a small contribution to the dDCS [see eqn (33) of ref. 4]. Notice that the uAiry + SC/N/PSA curve diverges as $\theta_{\mathrm{R}} \rightarrow 0^{\circ}$, the uBessel curve diverges as $\theta_{\mathrm{R}} \rightarrow \theta_{\mathrm{R}}^{\mathrm{r}}$, and the tAiry + SC/N/PSA curve diverges as $\theta_{\mathbf{R}} \rightarrow 180^{\circ}$.

Fig. 3(a) shows the range, $0^{\circ} \leq \theta_{\mathrm{R}} \leq 30^{\circ}$. We see that the PWS, uBessel and 6Hankel dDCSs agree to graphical accuracy, as does the uAiry + SC/N/PSA dDCS for $\theta_{\mathrm{R}} \gtrsim 2^{\circ}$. The same is mostly true for the range, $30^{\circ} \leq \theta_{\mathrm{R}} \leq 80^{\circ}$, displayed in Fig. 3(b), except near the maxima of the diffraction oscillations.

For $80^{\circ} \leq \theta_{\mathrm{R}} \leq \theta_{\mathrm{R}}^{\mathrm{r}}$, Fig. 3(c) shows that the uAiry + SC/N/PSA curve generally agrees best with the PWS dDCS, with the uBessel and 6 Hankel dDCSs being less accurate. This last result can be traced back to the 2Hankel approximation, which is generally less accurate than the uAiry approximation for the cuspoid test integrals in the Appendix. For $\theta_{\mathrm{R}} \gtrsim \theta_{\mathrm{R}}^{\mathrm{r}}$, the agreement between the PWS and tAiry + SC/N/PSA curves is satisfactory. As expected, the discrepancies between these two curves generally increases as we move further into the dark side of the rainbow.

Notice that the rainbow does not possess any supernumerary rainbows in the angular scattering; rather it is an example of a "broad rainbow"., 4,6,50

\section{VB. Nearside-farside analyses}

The NF decomposition of the PWS for $f\left(\theta_{\mathrm{R}}\right)$ is given by ref. 3,4 , $6,11,13-17$ and 19-24

$$
f\left(\theta_{\mathrm{R}}\right)=f^{(\mathrm{N})}\left(\theta_{\mathrm{R}}\right)+f^{(\mathrm{F})}\left(\theta_{\mathrm{R}}\right)
$$

where the $\mathrm{N}, \mathrm{F}$ subamplitudes are $\left(\theta_{\mathrm{R}} \neq 0, \pi\right)$

$$
f^{(\mathrm{N}, \mathrm{F})}\left(\theta_{\mathrm{R}}\right)=\frac{1}{2 \mathrm{i} k} \sum_{J=0}^{\infty}(2 J+1) \tilde{S}_{J} Q_{J}^{(\mathrm{N}, \mathrm{F})}\left(\cos \theta_{\mathrm{R}}\right)
$$

with

$$
Q_{J}^{(\mathrm{N}, \mathrm{F})}\left(\cos \theta_{\mathrm{R}}\right)=\frac{1}{2}\left[P_{J}\left(\cos \theta_{\mathrm{R}}\right) \pm \frac{2 \mathrm{i}}{\pi} Q_{J}\left(\cos \theta_{\mathrm{R}}\right)\right]
$$



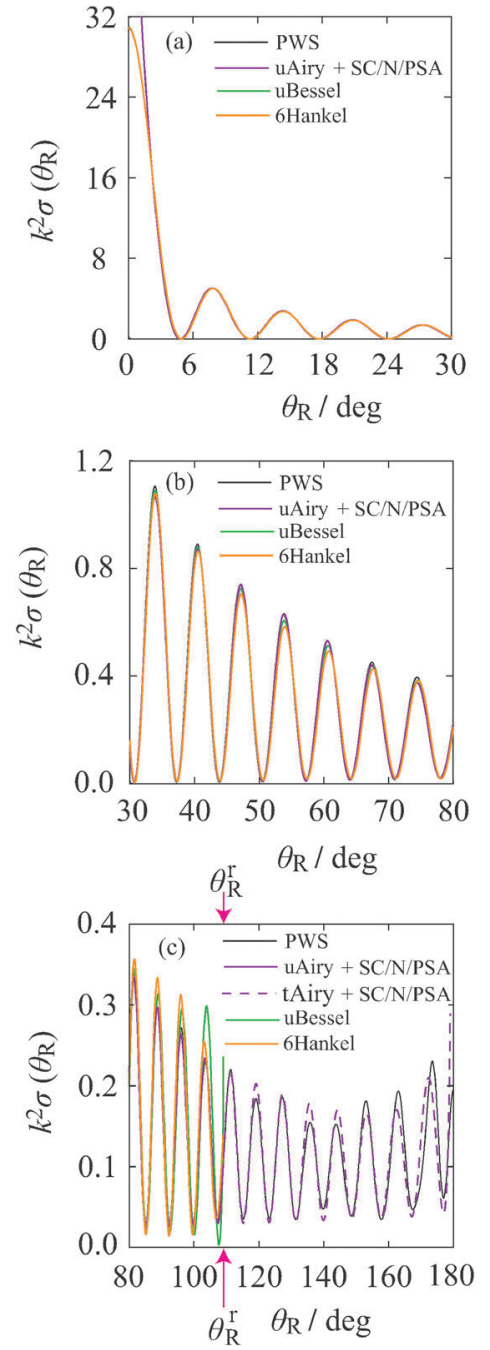

Fig. 3 Linear plot of the dimensionless angular distribution, $k^{2} \sigma\left(\theta_{R}\right)$ versus $\theta_{R}$. Black solid curve: PWS. Purple solid curve: uAiry + SC/N/PSA. Purple dashed curve: tAiry + SC/N/PSA. Green solid curve: uBessel. Orange solid curve: 6 Hankel. (a) $0^{\circ} \leq \theta_{\mathrm{R}} \leq 30^{\circ}$. The uAiry $+\mathrm{SC} / \mathrm{N} / \mathrm{PSA}$ curve diverges as $\theta_{\mathrm{R}} \rightarrow 0^{\circ}$. (b) $30^{\circ} \leq \theta_{\mathrm{R}} \leq 80^{\circ}$. The PWS and uAiry + SC/N/PSA curves are almost coincident for $\theta_{R} \gtrsim 35^{\circ}$. (c) $80^{\circ} \leq \theta_{R} \leq 180^{\circ}$. The pink solid arrows indicate the rainbow angle, $\theta_{\mathrm{R}}^{r}=109.2^{\circ}$. The uBessel curve diverges as $\theta_{\mathrm{R}} \rightarrow \theta_{\mathrm{R}}^{r}$. The tAiry + SC/N/PSA approximation is used for $\theta_{R} \geq \theta_{R}^{r}$; it diverges as $\theta_{R} \rightarrow 180^{\circ}$.

Eqn (68)-(70) are the Fuller decomposition for $f\left(\theta_{\mathrm{R}}\right){ }^{51}$ The corresponding N, F PWS DCSs are defined by

$$
\sigma^{(\mathrm{N}, \mathrm{F})}\left(\theta_{\mathrm{R}}\right)=\left|f^{(\mathrm{N}, \mathrm{F})}\left(\theta_{\mathrm{R}}\right)\right|^{2}
$$

In practice, we resum the PWS (46) three times $(r=3)$ before carrying out the NF decomposition because it is known this helps "clean" the N and F unresummed dDCSs and LAMs of unphysical structure. ${ }^{21-24}$ We then write $\mathrm{PWS} / \mathrm{N} / r=3$ and $\mathrm{PWS} / \mathrm{F} / r=3$ for the $\mathrm{N}$ and $\mathrm{F}$ resummed subamplitudes respectively. Totenhofer et $a .^{24}$ have presented a detailed account of resummation theory for a Legendre PWS; this theory is not repeated here.

Fig. 4(a) reports a NF analysis of the PWS dDCS. We see that the reaction is $\mathrm{N}$ dominant, although the $\mathrm{PWS} / \mathrm{F} / r=3 \mathrm{dDCS}$ is always significant, becoming increasingly important upon moving to smaller scattering angles. This results in pronounced
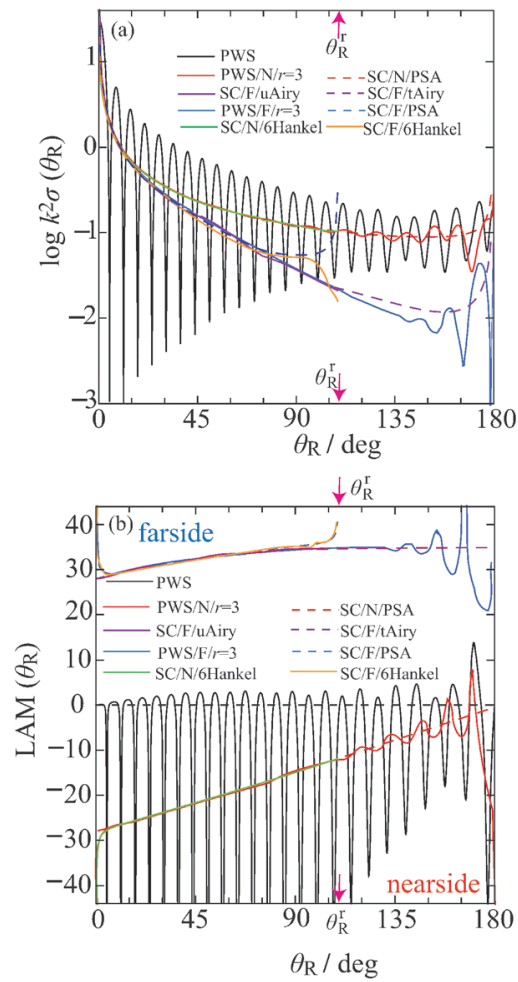

Fig. 4 Nearside-farside analyses for the dimensionless logarithmic angular distribution and the Local Angular Momentum. Black solid curve: PWS. Red solid curve: PWS/N/r = 3. Red dashed curve: SC/N/PSA. Solid purple curve: SC/F/uAiry. Purple dashed curve: SC/F/tAiry. Blue solid curve: $\mathrm{PWS} / \mathrm{F} / \mathrm{r}=3$. Blue dashed curve: SC/F/PSA. Green solid curve: SC/N/ 6 Hankel. Orange solid curve: SC/F/6 Hankel. The pink solid arrows indicate the rainbow angle, $\theta_{R}^{r}=109.2^{\circ}$. (a) Full and $N F \log k^{2} \sigma\left(\theta_{R}\right)$ versus $\theta_{R}$. (b) Full and $\mathrm{NF} \operatorname{LAM}\left(\theta_{\mathrm{R}}\right)$ versus $\theta_{\mathrm{R}}$.

diffraction oscillations in the full dDCS, which arise from interference between the $\mathrm{N}$ and $\mathrm{F}$ subamplitudes.

The PWS results in Fig. 3 for the dDCS, allowed us to test the accuracy of the semiclassical approximations. This is not the case for Fig. 4, because it is known that the NF PWS decomposition of eqn (68)-(71) is not unique: there is no guarantee that it will produce physically useful results. ${ }^{20-24}$ Rather we use the semiclassical approximations to provide a check on the physical effectiveness of the $\mathrm{N}, \mathrm{F} \mathrm{PWS} / r=3$ results. We also recall that the SC/F/6Hankel, SC/F/uAiry, SC/F/PSA and SC/N/6Hankel approximations are only defined for $\theta_{\mathrm{R}}<\theta_{\mathrm{R}}^{\mathrm{r}}$.

Fig. 4(a) plots the $\mathrm{N}$ and F dDCSs for the PWS $/ r=3$, PSA and 6 Hankel approximations, and the F dDCSs for the uAiry and tAiry approximations. The agreement between the semiclassical and $\mathrm{N}$, $\mathrm{F} \mathrm{PWS} / r=3 \mathrm{dDCSs}$ is seen to be generally good. The discrepancies are those expected from our earlier work. ${ }^{3,4,6,11,13-17,19-24}$ For example, the SC/F/PSA dDCS diverges as $\theta_{\mathrm{R}} \rightarrow \theta_{\mathrm{R}}^{\mathrm{r}}$, whilst the $\mathrm{N}$ and $\mathrm{F}$ PWS $/ r=3$ dDCSs exhibit unphysical oscillations at large angles.

Next we consider the NF analysis of the LAMs. ${ }^{21-24}$ The full LAM is defined by

$$
\operatorname{LAM}\left(\theta_{\mathrm{R}}\right)=\frac{\mathrm{d} \arg f\left(\theta_{\mathrm{R}}\right)}{\mathrm{d} \theta_{\mathrm{R}}}
$$


whilst the N, F LAMs are obtained from ref. 21-24

$$
\operatorname{LAM}^{(\mathrm{N}, \mathrm{F})}\left(\theta_{\mathrm{R}}\right)=\frac{\mathrm{d} \arg f^{(\mathrm{N}, \mathrm{F})}\left(\theta_{\mathrm{R}}\right)}{\mathrm{d} \theta_{\mathrm{R}}}
$$

In the PWS calculations, we again resum $f\left(\theta_{\mathrm{R}}\right)$ three times $(r=3)$ before making the NF decomposition. ${ }^{24}$ The args in eqn (72) and (73) are not necessarily principal values in order that the derivatives be well defined.

Fig. 4(b) shows full and N, F LAMs for the PWS $/ r=3$ and semiclassical approximations. We observe that the LAM information in Fig. 4(b) is consistent with the dDCS information in Fig. 4(a). The discrepancies between the N, F curves are similar to those we have seen previously, e.g., the unphysical oscillations in the N, F PWS $/ r=3$ LAMs as we approach the backward direction. ${ }^{21-24}$

We observe that the semiclassical $\mathrm{N}$ LAMs decrease in magnitude as $\theta_{\mathrm{R}}$ increases, and are similar to the LAM for the repulsive scattering of two hard spheres, ${ }^{23,24}$ i.e., the N scattering is direct. Next we examine the semiclassical F LAMs. We see that the semiclassical F LAMs are slowly increasing at small $\theta_{\mathrm{R}}$; then the SC/F/tAiry LAM becomes approximately constant at large angles, where it has the value 34.9 . In fact to a very good approximation we have (from ref. 4 and 6)

$$
\operatorname{LAM}_{\mathrm{tAiry}}^{(+)}\left(\theta_{\mathrm{R}}\right) \approx J_{\mathrm{r}}+1 / 2=35.0
$$

This behaviour of SC/F/tAiry corresponds in a Regge treatment to decaying (creeping) surface waves that propagate around the reaction zone (see also ref. 52.) When a single Regge pole $(n=0)$ dominates, we have (from ref. 6 and 27)

$$
\operatorname{LAM}_{\text {Regge }}^{(+)}\left(\theta_{\mathrm{R}}\right) \approx \operatorname{Re} J_{0}+1 / 2=34.9
$$

For a broad rainbow, we also expect $J_{\mathrm{r}} \approx \operatorname{Re} J_{0},{ }^{6,27}$ which is what we find. If we average over the oscillations in the PWS/F/ $r=3 \mathrm{LAM}$ for $100^{\circ} \leq \theta_{\mathrm{R}} \leq 165^{\circ}$ we obtain a mean value of 34.5 . Thus we have the result

$$
\begin{aligned}
\operatorname{LAM}_{r=3}^{(\mathrm{F})}\left(\theta_{\mathrm{R}}\right) & \approx \operatorname{LAM}_{\text {Regge }}^{(+)}\left(\theta_{\mathrm{R}}\right) \approx \operatorname{LAM}_{\mathrm{tAiry}}^{(+)}\left(\theta_{\mathrm{R}}\right) \approx \operatorname{Re} J_{0}+1 / 2 \\
& \approx J_{\mathrm{r}}+1 / 2
\end{aligned}
$$

This important result tells us that the PWS, semiclassical and Regge theories are all consistent with each other for the broad rainbow in Fig. 3 and 4(a).

\section{Conclusions}

In our recent paper with Zhang, ${ }^{6}$ we stated without proof the 6 Hankel asymptotic approximation for the scattering amplitude, which has the desirable property of being uniform for both a forward glory and a rainbow in the DCS of a chemical reaction. It is also a generic approximation. In this paper we have:

- Presented a detailed derivation of the 6Hankel approximation. Our derivation generalizes a method described by Carrier for an oscillating integral with two coalescing real stationary phase points. The generalization uses 3-jets of the phase at the stationary phase points, $x_{1}$ and $x_{2}$, followed by a discardation of the contributions from the unwanted stationary phase points, $u_{2}$ and $u_{1}$. Applying the resulting 2 Hankel approximation to the Legendre PWS for the scattering amplitude gives rise to the generic $6 \mathrm{Hankel}$ approximation. We also made a test of the accuracy of the $2 \mathrm{Hankel}$ approximation by applying it to three cuspoid oscillating integrals.

- Investigated some properties of the 6Hankel approximation. It has the advantage that each root contribution, $J_{1}, J_{2}, J_{3}$, appears separately in the 6Hankel expression, but has the disadvantage that third derivatives of arg $\tilde{S}(J)$ are required. In the limit $\theta_{\mathrm{R}} \rightarrow 0$, the 6 Hankel approximation reduces to the STA for describing the glory. And in the limit $\theta_{\mathrm{R}} \rightarrow \theta_{\mathrm{R}}^{\mathrm{r}}$, we obtain the tAiry approximation for rainbow scattering.

- Assessed the accuracy of the 6Hankel approximation for $\theta_{\mathrm{R}}<\theta_{\mathrm{R}}^{\mathrm{r}}$. Using a $J$-shifted Eckart parametrization of the $\mathrm{S}$ matrix, we found that both the 6Hankel and uBessel DCSs agreed well with the PWS DCS at angles close to the forward direction. However using numerical $S$ matrix data, we earlier had found ${ }^{6}$ the 6 Hankel DCS to be less accurate than the uBessel DCS at small angles - probably because of the difficulty of accurately calculating the $\tilde{\Theta}^{\prime \prime}\left(J_{i}\right)$. Near the rainbow angle, the 6 Hankel DCS generally exhibited greater deviations from the PWS DCS compared to the uAiry + SC/N/PSA DCS. This can be traced back to the 2 Hankel approximation, which was generally less accurate than the uAiry approximation for the cuspoid test integrals.

The above trends can be understood in a more general way by remembering that the phases of the semiclassical integrands are not approximated in the uBessel and uAiry approximations rather exact local one-to-one transformations are made - and only the pre-exponential factors are approximated. ${ }^{3,4,7,8}$ Whereas, in the 2Hankel and 6Hankel approximations, both the phases and pre-exponential factors are approximated.

Also relevant is the following comment made by Ursell in his last published paper concerning the solution of (water) wave problems: ${ }^{53}$

"Such a problem is usually solved by applying a sequence of mathematical arguments, and it would be helpful if some or all of the successive steps in this sequence could be given a physical interpretation. In the author's experience this is generally not possible."

Ursell illustrates his comment by several examples of mathematical steps that do not have a physical interpretation, in particular the use of the exact local one-to-one transformation employed in the method of Chester et al. ${ }^{39}$ for the uniform asymptotic evaluation of an oscillating integral with two coalescing saddle points. This is also the key transformation used in the derivation of the uAiry approximation. ${ }^{4,8}$

\section{Appendix: application of the $2 \mathrm{Hankel}$ approximation to cuspoid integrals}

In this Appendix, we assess the accuracy of the 2Hankel approximation (34) when it is applied to three cuspoid integrals ${ }^{28,29}$ of the Case A type. We also compare with results from the 
uniform Airy approximation (uAiry) derived by $\mathrm{CM},{ }^{8}$ which is based on the technique of Chester et al. ${ }^{39}$ For a Case A integral, the uniform Airy approximation is given in Section IIIG of CM, namely

$$
\begin{aligned}
I_{\text {uAiry }}(\alpha)= & 2^{1 / 2} \pi \exp (\mathrm{i} A)\left\{\left[\frac{g_{1}}{\left(-f_{1}{ }^{\prime \prime}\right)^{1 / 2}}+\frac{g_{2}}{\left(f_{2}{ }^{\prime \prime}\right)^{1 / 2}}\right] \varsigma^{1 / 4} \mathrm{Ai}(-\varsigma)\right. \\
& \left.+\mathrm{i}\left[\frac{g_{1}}{\left(-f_{1}^{\prime \prime}\right)^{1 / 2}}-\frac{g_{2}}{\left(f_{2}{ }^{\prime \prime}\right)^{1 / 2}}\right] \varsigma^{-1 / 4} \mathrm{Ai}^{\prime}(-\varsigma)\right\}
\end{aligned}
$$

where

$$
A(\alpha)=\frac{1}{2}\left(f_{1}+f_{2}\right)
$$

and

$$
\varsigma(\alpha)=\left[\frac{3}{4}\left(f_{1}-f_{2}\right)\right]^{2 / 3}
$$

Here $\operatorname{Ai}^{\prime}(x)$ means $\operatorname{dAi}(x) / d x$. Note that $\varsigma(\alpha) \geq 0$ for real roots. When $\varsigma \gg 1$, so the stationary phase points are well separated, the simple stationary phase result (2) is obtained upon replacing $\mathrm{Ai}(-\varsigma)$ and $\mathrm{Ai}^{\prime}(-\varsigma)$ by their asymptotic approximations. When $\varsigma \approx 0$, so the stationary phase points are close together, the transitional Airy approximation (4) is obtained when $f(\alpha ; x)$ is approximated by its 3 -jet, as in eqn (3) or (6). For this situation, eqn (A1) for $I_{\mathrm{uAiry}}(\alpha)$ becomes an exact result, provided $g(x)=1$.

In the following three examples, all the phases are real and have a linear dependence on $\alpha$ of the type, $-\alpha x$. In all three cases, $g(x)=1$. The corresponding oscillating integrals have been calculated numerically by deforming the contour of integration from the real axis into the complex plane as explained in ref. $54-56$.

\section{Example 1. Cubic polynomial phase}

We write the cubic phase in the form

$$
f_{1}(\alpha ; x)=-\alpha x+a_{2} \frac{x^{2}}{2}+a_{3} \frac{x^{3}}{3}
$$

where $\alpha, a_{2}, a_{3}$ are real numbers chosen so that $f_{1}(\alpha ; x)$ has two real stationary phase points [i.e., real roots of $\mathrm{d} f_{1}(\alpha ; x) / \mathrm{d} x=0$ ], which can coalesce as $\alpha$ varies; for example, $a_{2}=1, a_{3}=1, \alpha \geq$ $-1 / 4$. More generally, we require the discriminant, $D_{1}$, of the quadratic equation $\mathrm{d} f_{1}(\alpha ; x) / \mathrm{d} x=0$, namely $D_{1}=a_{2}{ }^{2}+4 \alpha a_{3}$, to satisfy $D_{1}>0$ for separate real roots or $D_{1}=0$ for one real double root. Then $I_{2 \mathrm{H}}(\alpha), I_{\mathrm{uAiry}}(\alpha)$ and $I_{\text {tAiry }}(\alpha)$ are exact results for the cubic phase (A2), which is useful for the checking of computer programs.

\section{Example 2. Quintic polynomial phase: an oddoid integral of order two}

In this example we choose

$$
f_{2}(\alpha ; x)=-\alpha x+a_{3} \frac{x^{3}}{3}+a_{5} \frac{x^{5}}{5}
$$

where $a_{3}>0, a_{5}>0$ and $\alpha \geq 0$. (For the tAiry approximation we also let $\alpha<0$.) Since $f_{2}(\alpha ; x)$ is an odd function of $x, I_{2}(\alpha)$ is purely real; it is an example of an oddoid integral of order two. ${ }^{29}$ We have

$$
\begin{aligned}
I_{2}(\alpha) & =\int_{-\infty}^{\infty} \exp \left[\mathrm{i} f_{2}(\alpha ; x)\right] \mathrm{d} x=2 \int_{0}^{\infty} \cos \left[f_{2}(\alpha ; x)\right] \mathrm{d} x \\
& =\frac{1}{a_{5}^{1 / 5}} O_{2}\left(\frac{-\alpha}{a_{5}^{1 / 5}}, \frac{a_{3}}{a_{5}^{3 / 5}}\right)
\end{aligned}
$$

where the oddoid integral is defined in ref. 29

$$
\begin{aligned}
O_{2}\left(b_{1}, b_{2}\right) & =\int_{-\infty}^{\infty} \exp \left[\mathrm{i}\left(b_{1} t+b_{2} \frac{t^{3}}{3}+\frac{t^{5}}{5}\right)\right] \mathrm{d} t \\
& =2 \int_{0}^{\infty} \cos \left(b_{1} t+b_{2} \frac{t^{3}}{3}+\frac{t^{5}}{5}\right) \mathrm{d} t
\end{aligned}
$$

with $b_{1}$ and $b_{2}$ real. Hobbs et al. ${ }^{29}$ have discussed the properties of oddoid (and evenoid) integrals. Now Descartes' Rule of Signs ${ }^{57}$ tells us that the quartic equation $\mathrm{d} f_{2}(\alpha ; x) / \mathrm{d} x=0$ for $\alpha>0$ has one positive root and one negative root, which coalesce when $\alpha=0$. For $\alpha<0$, these roots become complex conjugates.

Fig. 5(a) plots $I_{2}(\alpha)$ versus $\alpha$ for the range $0 \leq \alpha \leq 15$ with phase parameters of $a_{3}=a_{5}=5$. The oscillatory nature of $I_{2}(\alpha)$ can be clearly seen. Also plotted are the 2 Hankel and uAiry approximations as well as the SPA [these curves are also drawn in more detail for $\alpha \leq 5$ in Fig. 5(b)]. The SPA is accurate for $\alpha \gtrsim 2$ and, as expected, it diverges as $\alpha \rightarrow 0$. The 2Hankel approximation becomes systematically smaller than $I_{2}(\alpha)$ for $\alpha \lesssim 3$,
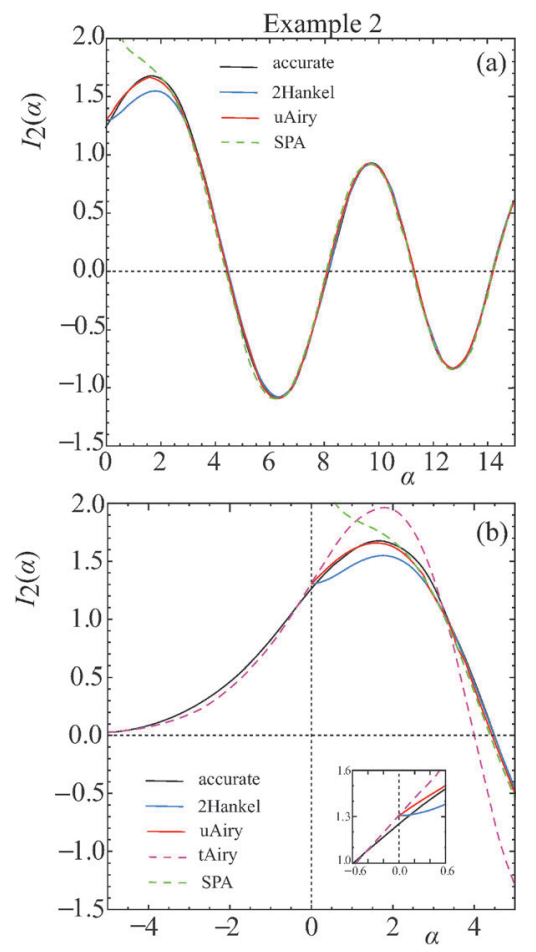

Fig. 5 (a) $I_{2}(\alpha)$ and three asymptotic approximations for $0 \leq \alpha \leq 15$. Black solid curve: $I_{2}(\alpha)$. Red solid curve: UAiry. Blue solid curve: 2 Hankel. Green dashed curve: SPA. (b) $I_{2}(\alpha)$ and four asymptotic approximations for $-5 \leq \alpha \leq 5$. Black solid curve: $I_{2}(\alpha)$. Red solid curve: uAiry. Blue solid curve: 2 Hankel. Green dashed curve: SPA. Pink dashed curve: tAiry. The inset shows the corresponding curves for $-0.6 \leq \alpha \leq 0.6$ (not SPA). 
until $\alpha \approx 0.15$, when the two curves cross. The uAiry approximation agrees closely with $I_{2}(\alpha)$ over the whole range of $\alpha$.

It was mentioned in Section IIB that the tAiry approximation is also valid for negative $\alpha$. Fig. 5 (b) compares $I_{\text {tAiry }}(\alpha)$ with $I_{2}(\alpha)$ for $-5 \leq \alpha \leq 5$. It can be seen that there is good agreement between the two curves for negative $\alpha$. This finding is very useful since the theory and application of the 2Hankel and uAiry approximations for negative $\alpha$ in practical applications is usually much more difficult than for $\alpha>0$. However Fig. 5(b) shows that the tAiry approximation quickly becomes inaccurate for positive $\alpha$, in particular for the amplitude of the oscillation at $\alpha \approx 2$. And this continues to be the case for $\alpha>5$ where the positions and amplitudes of the maxima and minima in $I_{2}(\alpha)$ are not reproduced (not shown). At $\alpha=0$, the 6 Hankel and uAiry approximations become equivalent to the tAiry approximation; this result can be seen visually in the inset to Fig. 5(b). Note that the tAiry approximation is obtained by putting $a_{5}=0$ in the phase (A3). Also from eqn (5) we have

$$
I_{\mathrm{tAiry}}(\alpha=0)=\frac{2 \pi}{(45)^{1 / 3} \Gamma(2 / 3)}=1.305
$$

The accurate value for $I_{2}(\alpha=0)$ is 1.251 , so the percentage error in $I_{\text {tAiry }}(\alpha=0)$ is $4.3 \%$.

\section{Example 3. Quintic polynomial phase: A swallowtail integral}

In our third example we choose

$$
f_{3}(\alpha ; x)=-\alpha x+a_{3} \frac{x^{3}}{3}+a_{4} \frac{x^{4}}{4}+a_{5} \frac{x^{5}}{5}
$$

with $a_{3}>0, a_{4}>0, a_{5}>0$ and $\alpha \geq 0$. (Again we also let $\alpha<0$ for the tAiry approximation.) The corresponding oscillating integral

$$
I_{3}(\alpha)=\int_{-\infty}^{\infty} \exp \left[\mathrm{i} f_{3}(\alpha ; x)\right] \mathrm{d} x
$$

is an example of a swallowtail integral, ${ }^{28,55}$ which is complex valued in general. Descartes' Rule of Signs ${ }^{57}$ tells us that the quartic equation $\mathrm{d} f_{3}(\alpha ; x) / \mathrm{d} x=0$ for $\alpha>0$ has one positive root and one negative root, which coalesce at $\alpha=0$. For $\alpha<0$, these roots become complex conjugates. We have compared $I_{2 \mathrm{H}}(\alpha)$, $I_{\text {uAiry }}(\alpha), I_{\text {tAiry }}(\alpha)$ with $I_{3}(\alpha)$ for many values of $a_{3}, a_{4}, a_{5}$ when $0 \leq \alpha \leq 20$ [and also $-5 \leq \alpha \leq 20$ for the tAiry approximation]. In general, the accuracy of the asymptotic formulae are similar to that already discussed in Fig. 5 for Example 2, so, we do not display the corresponding graphs.

\section{Acknowledgements}

Support of this research by the UK Engineering and Physical Sciences Research Council and a UK Leverhulme Trust Emeritus Fellowship to JNLC is gratefully acknowledged.

\section{References}

1 Tutorials in Molecular Reaction Dynamics, ed. M. Brouard and C. Vallance, RSC Publishing, Cambridge, UK, 2010.
2 D. M. Neumark, A. M. Wodtke, G. N. Robinson, C. C. Hayden and Y. T. Lee, J. Chem. Phys., 1985, 82, 3045.

3 J. N. L. Connor, Phys. Chem. Chem. Phys., 2004, 6, 377.

4 C. Xiahou and J. N. L. Connor, J. Phys. Chem. A, 2009, 113, 15298.

5 X. Wang, W. Dong, M. Qiu, Z. Ren, L. Che, D. Dai, X. Wang, X. Yang, Z. Sun, B. Fu, S.-Y. Lee, X. Xu and D. H. Zhang, Proc. Natl. Acad. Sci. U. S. A., 2008, 105, 6227.

6 C. Xiahou, J. N. L. Connor and D. H. Zhang, Phys. Chem. Chem. Phys., 2011, 13, 12981. In Section VA.4, for "displaced from $\theta_{\mathrm{R}}=10^{\circ}$ ", read "displaced from $\theta_{\mathrm{R}}=0^{\circ}$ ",

7 J. N. L. Connor, Mol. Phys., 2005, 103, 1715.

8 J. N. L. Connor and R. A. Marcus, J. Chem. Phys., 1971, 55, 5636; For a commentary, see: J. N. L. Connor, Current Contents: Physical, Chemical and Earth Sciences, 1991, vol. 31, no. 50, p. 10; J. N. L. Connor, Current Contents: Engineering, Technology and Applied Sciences, 1991, vol. 22, no. 50, p. 10. Also available at http:/garfield.library.upenn.edu/classics1991/ A1991GR14400001.pdf.

9 W. H. Miller, J. Chem. Phys., 1968, 48, 464. In eqn (6), for “ $H_{0}^{\left(n_{i}\right)} \times\left[\left(l_{i}+\frac{1}{2}\right) \theta\right]$ ”, read “ $H_{0}^{\left(n_{i}\right)}\left[\left(l_{i}+\frac{1}{2}\right) \theta\right]$ ”.

10 D. Sokolovski, Chem. Phys. Lett., 2003, 370, 805.

11 C. Xiahou and J. N. L. Connor, Mol. Phys., 2006, 104, 159.

12 J. A. Adam, Phys. Rep., 2002, 356, 229.

13 C. Xiahou and J. N. L. Connor, in Semiclassical and Other Methods for Understanding Molecular Collisions and Chemical Reactions, ed. S. Sen, D. Sokolovski and J. N. L. Connor, Collaborative Computational Project on Molecular Quantum Dynamics (CCP6), Daresbury Laboratory, Warrington, UK, 2005, pp. 44-49, ISBN 0-9545289-3-X.

14 J. N. L. Connor, X. Shan, A. J. Totenhofer and C. Xiahou, in Multidimensional Quantum Mechanics with Trajectories, ed.

D. V. Shalashilin and M. P. de Miranda, Collaborative Computational Project on Molecular Quantum Dynamics (CCP6), Daresbury Laboratory, Warrington, UK, 2009, pp. 38-47, ISBN 978-0-9545289-8-0.

15 X. Shan and J. N. L. Connor, Phys. Chem. Chem. Phys., 2011, 13, 8392.

16 X. Shan and J. N. L. Connor, J. Chem. Phys., 2012, 136, 044315.

17 X. Shan and J. N. L. Connor, J. Phys. Chem. A, 2012, 116, 11414.

18 G. F. Carrier, J. Fluid Mech., 1966, 24, 641. In the Appendix, p. 658: On line 7, for " $h(z)=\alpha$ ”, read " $h^{\prime}(z)=\alpha$ ”; on line 17 , for " $f$ " $\left(z_{0}\right)$ ". read " $h$ " $\left(z_{0}\right)$ "; on line 26 , for eqn (1)", read "eqn (A1)"; in eqn (A5), for " $f^{\prime \prime \prime}\left(z_{0}\right)$ ", $\operatorname{read}$ " $h^{\prime \prime \prime}\left(z_{0}\right)$ ". On p. 659 , in eqn (A6), for " $f^{\prime \prime \prime}\left(z_{0}\right)$ ", read " $h^{\prime \prime \prime}\left(z_{0}\right)$ ".

19 J. N. L. Connor, P. McCabe, D. Sokolovski and G. C. Schatz, Chem. Phys. Lett., 1993, 206, 119.

20 P. McCabe and J. N. L. Connor, J. Chem. Phys., 1996, 104, 2297.

21 R. Anni, J. N. L. Connor and C. Noli, Phys. Rev. C: Nucl. Phys., 2002, 66, 044610.

22 R. Anni, J. N. L. Connor and C. Noli, Khim. Fiz., 2004, 23(2), 6. Also available at: http://arXiv.org/abs/physics/0410266. 
23 J. N. L. Connor and R. Anni, Phys. Chem. Chem. Phys., 2004, 6, 3364.

24 A. J. Totenhofer, C. Noli and J. N. L. Connor, Phys. Chem. Chem. Phys., 2010, 12, 8772.

25 J. N. L. Connor, J. Chem. Soc., Faraday Trans., 1990, 86, 1627.

26 D. Sokolovski, J. F. Castillo and C. Tully, Chem. Phys. Lett., 1999, 313, 225.

27 J. N. L. Connor, J. Chem. Phys., 2013, 138, 124310.

28 J. N. L. Connor, Mol. Phys., 1976, 31, 33.

29 C. A. Hobbs, J. N. L. Connor and N. P. Kirk, J. Comput. Appl. Math., 2007, 207, 192. In eqn (21), in the $T_{5}(t, a)$ expression, for " $4 a t^{3}$ ", read " $5 a t^{3}$ ".

30 W. H. Miller, J. Chem. Phys., 1970, 53, 3578.

31 J. N. L. Connor, Faraday Discuss. Chem. Soc., 1973, 55, 51.

32 J. N. L. Connor, Mol. Phys., 1973, 25, 181.

33 J. N. L. Connor, Mol. Phys., 1973, 26, 1217.

34 J. N. L. Connor, Mol. Phys., 1973, 26, 1371.

35 J. N. L. Connor, Mol. Phys., 1974, 27, 853.

36 J. N. L. Connor, Chem. Phys. Lett., 1974, 25, 611.

37 D. Sokolovski, J. N. L. Connor and G. C. Schatz, Chem. Phys. Lett., 1995, 238, 127.

38 D. Sokolovski, J. N. L. Connor and G. C. Schatz, J. Chem. Phys., 1995, 103, 5979.

39 C. Chester, B. Friedman and F. Ursell, Proc. Cambridge Philos. Soc., 1957, 53, 599; Reprinted in Ship Hydrodynamics, Water Waves and Asymptotics, Collected Papers of F. Ursell, 1946-1992, ed. F. Ursell, World Scientific, Singapore, 1994, vol. II, pp. 708-720; For a commentary, see Ship Hydrodynamics, Water Waves and Asymptotics, Collected Papers of F. Ursell, 1946-1992, ed. F. Ursell, World Scientific, Singapore, 1994, vol. II, p. 570.

40 T. Poston and I. N. Stewart, Taylor Expansions and Catastrophes, Pitman Publishing, London, UK, 1976, p. 13.

41 W. Sanns, Catastrophe Theory with Mathematica: A Geometric Approach, Der Andere Verlag, Osnabrück, Germany, 2000, ch. 3.

42 F. W. J. Olver, in NIST Handbook of Mathematical Functions, ed. F. W. J. Olver, D. W. Lozier, R. F. Boisvert and C. W. Clark, Cambridge University Press, Cambridge, UK, 2010, ch. 9, item 9.6.6. Also available at http://dlmf.nist.gov/, Release 1.0.6 of 2013-05-06.
43 F. W. J. Olver and L. C. Maximon, in NIST Handbook of Mathematical Functions, ed. F. W. J. Olver, D. W. Lozier, R. F. Boisvert and C. W. Clark, Cambridge University Press, Cambridge, UK, 2010, ch. 10, items 10.2.5 and 10.2.6. Also available at http://dlmf.nist.gov/, Release 1.0.6 of 2013-05-06.

44 F. W. J. Olver and L. C. Maximon, in NIST Handbook of Mathematical Functions, ed. F. W. J. Olver, D. W. Lozier, R. F. Boisvert and C. W. Clark, Cambridge University Press, Cambridge, UK, 2010, ch. 10, item 10.7.7. Also available at http://dlmf.nist.gov/, Release 1.0.6 of 2013-05-06.

45 E. Hilb, Math. Z., 1919, 5, 17.

46 N. M. Temme, Special Functions: An Introduction to the Classical Functions of Mathematical Physics, Wiley, New York, USA, 1996, p. 160.

47 F. W. J. Olver and L. C. Maximon, in NIST Handbook of Mathematical Functions, ed. F. W. J. Olver, D. W. Lozier, R. F. Boisvert and C. W. Clark, Cambridge University Press, Cambridge, UK, 2010, ch. 10, item 10.4.4. Also available at http://dlmf.nist.gov/, Release 1.0.6 of 2013-05-06.

48 D. Sokolovski and A. Z. Msezane, Phys. Rev. A: At., Mol., Opt. Phys., 2004, 70, 032710.

49 P. D. D. Monks, C. Xiahou and J. N. L. Connor, J. Chem. Phys., 2006, 125, 133504.

50 J. N. L. Connor, D. Farrelly and D. C. Mackay, J. Chem. Phys., 1981, 74, 3278.

51 R. C. Fuller, Phys. Rev. C: Nucl. Phys., 1975, 12, 1561.

52 D. Sokolovski, D. De Fazio, S. Cavalli and V. Aquilanti, Phys. Chem. Chem. Phys., 2007, 9, 5664.

53 F. Ursell, J. Eng. Math., 2007, 58, 7.

54 J. N. L. Connor and P. R. Curtis, J. Phys. A: Math. Gen., 1982, 15, 1179.

55 J. N. L. Connor, P. R. Curtis and D. Farrelly, J. Phys. A: Math. Gen., 1984, 17, 283.

56 N. P. Kirk, J. N. L. Connor, P. R. Curtis and C. A. Hobbs, Comput. Phys. Commun., 2000, 132, 142.

57 J. V. Uspensky, Theory of Equations, McGraw-Hill, New York, USA, 1963, paperback version of the 1948 edition, ch. VI, Section 10. 